\title{
RANGES OF PERTURBED MAXIMAL MONOTONE AND $m$-ACCRETIVE OPERATORS IN BANACH SPACES
}

\author{
ZHENGYUAN GUAN AND ATHANASSIOS G. KARTSATOS
}

\begin{abstract}
A more comprehensive and unified theory is developed for the solvability of the inclusions $S \subset \overline{R(A+B)}$, int $S \subset R(A+B)$, where $A: X \supset$ $D(A) \rightarrow 2^{Y}, B: X \supset D(B) \rightarrow Y$ and $S \subset X$. Here, $X$ is a real Banach space and $Y=X$ or $Y=X^{*}$. Mainly, $A$ is either maximal monotone or $m$ accretive, and $B$ is either pseudo-monotone or compact. Cases are also considered where $A$ has compact resolvents and $B$ is continuous and bounded. These results are then used to obtain more concrete sets in the ranges of sums of such operators $A$ and $B$. Various results of Browder, Calvert and Gupta, Gupta, Gupta and Hess, and Kartsatos are improved and/or extended. The methods involve the application of a basic result of Browder, concerning pseudo-monotone perturbation.' of maximal monotone operators, and the Leray-Schauder degree theory.
\end{abstract}

\section{INTRODUCTION-PRELIMINARIES}

In what follows, $X$ is a real Banach space with dual space $X^{*}$ and normalized duality mapping $J$. We denote the norms of $X$ and $X^{*}$ by $\|\cdot\|$. For $x \in X$ and $x^{*} \in X^{*}$, we use the symbol $\left(x^{*}, x\right)$ or the symbol $\left(x, x^{*}\right)$ to denote the value of $x^{*}$ at $x$. Let $Y$ be another real Banach space. Unless otherwise stated, or implied, the term "continuous" means strongly continuous. An operator $T: X \supset D(T) \rightarrow Y$, is "demicontinuous" if it is continuous from the strong topology of $X$ to the weak topology of $Y$. It is "completely continuous" if it is continuous from the weak topology of $X$ to the strong topology of $Y$. It is "compact" if it is continuous and maps bounded subsets of $D(T)$ onto relatively compact sets of $Y$.

A mapping $T: X \supset D(T) \rightarrow 2^{X^{*}}$ is said to be "monotone" if for every $x, y \in D(T), u \in T x$ and $v \in T y$ we have

$$
(u-v, x-y) \geq 0 .
$$

A monotone operator $T$ is "strictly monotone" if (1.1) holds with a strict inequality whenever $x \neq y$. It is "strongly monotone" if there exists a positive constant $\alpha$ such that (1.1) holds with 0 replaced by $\alpha\|x-y\|^{2}$.

Received by the editors November 30, 1993.

1991 Mathematics Subject Classification. Primary 47H17; Secondary 47B07, 47H05, 471106, $47 \mathrm{H} 10$.

Key words and phrases. Maximal monotone operator, pseudo-monotone operator, $m$-accretive operator, compact perturbation, compact resolvent, range of sums. 
A monotone mapping $T: X \supset D(T) \rightarrow 2^{X^{*}}$ is said to be "maximal monotone", if $R(T+\lambda J)=X^{*}$ for every $\lambda>0$. This is equivalent to saying that: $T$ is monotone and

$$
\left(u-u_{0}, x-x_{0}\right) \geq 0
$$

for every $x \in D(T)$ and $u \in T x$ implies $x_{0} \in D(T)$ and $u_{0} \in T x_{0}$.

A mapping $T: X \supset D(T) \rightarrow X^{*}$ is said to be "pseudo-monotone" if for every sequence $\left\{x_{n}\right\} \subset D(T)$ with $x_{n} \rightarrow x \in D(T)$ and $\limsup _{n \rightarrow \infty}\left(T x_{n}, x_{n}-x\right) \leq$ 0 , we have

$$
(T x, x-y) \leq \liminf _{n \rightarrow \infty}\left(T x_{n}, x_{n}-y\right)
$$

for every $y \in D(T)$. A mapping $T: X \supset D(T) \rightarrow X^{*}$ is said to be "generalized pseudo-monotone" if for every sequence $\left\{x_{n}\right\} \subset D(T)$ with $x_{n} \rightarrow x \in$ $D(T), T x_{n}-y$ and $\limsup _{n \rightarrow \infty}\left(T x_{n}, x_{n}-x\right) \leq 0$, we have $y=T x$ and $\left(T x_{n}, x_{n}\right) \rightarrow(T x, x)$. It is said to be of "type $\left(S_{+}\right)$" if for every sequence $\left\{x_{n}\right\} \subset D(T)$ with $x_{n} \rightarrow x \in X$ and $\lim _{\sup _{n \rightarrow \infty}}\left(T x_{n}, x_{n}-x\right) \leq 0$, we have $x_{n} \rightarrow x$. It is said to be of "type $(M)$ " if for any sequence $\left\{x_{n}\right\} \subset D(T)$ with $x_{n} \rightarrow x \in D(T), T x_{n}-y$ and $\lim _{\sup _{n \rightarrow \infty}}\left(T x_{n}, x_{n}-x\right) \leq 0$, we have $T x=y$. For the basic properties of mappings of monotone type and the duality mapping $J$, we refer the reader to Barbu [1], Browder [6], Cioranescu [9], Pascali and Sburlan [23] and Zeidler [25]. For Hilbert spaces $H$, the book of Brézis [2] is also a classical reference.

An operator $T: X \supset D(T) \rightarrow 2^{X}$ is called "accretive" if for every $x, y \in$ $D(T)$ there exists $j \in J(x-y)$ such that

$$
(u-v, j) \geq 0
$$

for all $u \in T x, v \in T y$. An accretive operator $T$ is " $m$-accretive", if $R(T+\lambda I)=X$ for all $\lambda \in(0, \infty)$.

For an $m$-accretive operator $T$, the "resolvents" $J_{\lambda}: X \rightarrow D(T)$ of $T$ are defined by $J_{\lambda}=(I+\lambda T)^{-1}$ for all $\lambda \in(0, \infty)$. The "Yosida approximants" $T_{\lambda}: X \rightarrow X$ of $T$ are defined by $T_{\lambda}=\frac{1}{\lambda}\left(I-J_{\lambda}\right)$.

Some of the main properties of $J_{\lambda}$ and $T_{\lambda}$ are given below:

1. $\left\|J_{\lambda} x-J_{\lambda} y\right\| \leq\|x-y\|$ for all $x, y \in X$.

2. $\left\|J_{\lambda} x-x\right\|=\lambda\left\|T_{\lambda} x\right\| \leq \lambda \inf \{\|y\| ; y \in T x\}$ for all $x \in D(T)$.

3. $T_{\lambda}$ is $m$-accretive on $X$ and $\left\|T_{\lambda} x-T_{\lambda} y\right\| \leq \frac{2}{\lambda}\|x-y\|$ for all $\lambda>0$, $x, y \in X$.

4. $T_{\lambda} x \in T J_{\lambda} x$ for all $x \in X$.

We refer the reader to the books of Barbu [1], Browder [6], Cioranescu [9] and Lakshmikantham and Leela [21] for facts about accretive operators. For a survey article on compact perturbations and compact resolvents of accretive operators, we cite Kartsatos' paper [16]. We denote by $B_{r}(0)$ the open ball of $X$ or $X^{*}$ with center at zero and radius $r>0$.

The purpose of this paper is to present several range results for various perturbations of maximal monotone and $m$-accretive operators. These results are of the type $S \subset \overline{R(A+B)}$ and int $S \subset R(A+B)$, where $S$ is a fixed subset of $X$ and $A, B$ are (possibly nonlinear) operators with $A$ maximal monotone or $m$-accretive. In Section 2 of the paper we study such problems in which $A$ is a maximal monotone operator and $B$ pseudo-monotone. These results are based 
on a very general result of Browder (see Theorem A), involving finitely continuous pseudo-monotone perturbations of maximal monotone operators. Section 3 of the paper is mainly devoted to compact perturbations $B$ of maximal monotone operators $A$, as well as bounded and continuous perturbations $B$ of maximal monotone operators $A$ that have compact resolvents. These results are generalizing and improving certain ideas of Kartsatos [18] by using in part homotopies introduced in [17]. Kartsatos studied in [18] the above problem, in the " $m$-accretive" case, with $S=S_{1}+S_{2}$, where the sets $S_{i}, i=1,2$, satisfied two separate conditions involving the operators $A$ and $B$. Here, we have managed to replaced these two conditions by a single one, improving thus the relevant results of [18] in the case of maximal monotone operators $A$. Numerous results are thus extended and/or improved due to Brézis and Haraux [4], Brézis and Nirenberg [5], Browder [7], Gupta [13] and Gupta and Hess [14]. In Section 4, we obtain results for perturbations of $m$-accretive operators which parallel those of Section 3. Our results of Section 4 provide very general "inner product" conditions for the existence of zeros of $m$-accretive operators. We have thus answered in the affirmative a problem stated by Kartsatos in the discussion of [18], to the effect that the methods of ranges of sums can actually be used in order to improve results involving the existence of zeros of perturbations of accretive operators.

The following lemma is due to Browder [7, Lemma 1].

Lemma 1.1. Let $X$ be a Banach space, $\left\{x_{n}\right\}$ a sequence in $X,\left\{\alpha_{n}\right\}$ a sequence of positive constants with $\alpha_{n} \rightarrow 0$ as $n \rightarrow \infty$. Fix $r>0$ and assume that for every $h \in X^{*}$ with $\|h\| \leq r$ there exists a constant $C_{h}$ such that $\left(h, x_{n}\right) \leq$ $\alpha_{n}\left\|x_{n}\right\|+C_{h}$, for all $n$. Then the sequence $\left\{x_{n}\right\}$ is bounded.

Lemma 1.2 below follows easily from Browder's Lemma 2 in [7].

Lemma 1.2. Let $\left\{x_{n}\right\} \subset X$ and $\left\{\alpha_{n}\right\}$ a sequence of positive numbers tending to zero as $n \rightarrow \infty$. Fix $r>0$ and assume that for every $h \in X$ with $\|h\| \leq r$ there exists a constant $C_{h}$ such that

$$
\langle h, j\rangle \leq \alpha_{n}\left\|x_{n}\right\|+C_{h}
$$

for all $n$ and some $j=j_{n} \in J x_{n}$. Then the sequence $\left\{x_{n}\right\}$ is bounded.

The next lemma is due to Prüß [24].

Lemma 1.3. A Banach space $X$ is uniformly convex if and only if for each $r>0$ there exists a non-decreasing function $\omega_{r}: R_{+} \rightarrow R_{+}$such that $\omega_{r}(\rho)>0$ for $\rho>0, \omega_{r}(0)=0$ and

$$
\left(u^{*}-v^{*}, x-y\right) \geq \omega_{r}(\|x-y\|)\|x-y\|
$$

for all $x, y \in \overline{B_{r}(0)}, u^{*} \in J x, v^{*} \in J y$.

\section{PSEUdo-MONOTONE PERTURBATIONS OF MAXIMAL MONOTONE OPERATORS}

We denote by $\Gamma$ the set of all functions $\beta: R^{+} \rightarrow R^{+}$such that $\beta(r) \rightarrow 0$ as $r \rightarrow \infty$. For pseudo-monotone perturbations $B$ of maximal monotone operators $A$, we have the following fundamental result which is due to Browder (cf. Pascali and Sburlan [23, Theorem on p. 120] and Zeidler [25, Theorem 32.A]). This result is an extension to infinite dimensional spaces of the wellknown Debrunner-Flor lemma. 
Theorem A. Let $X$ be reflexive and $C$ a nonempty closed, convex set in $X$. Let $A: D(A) \subset C \rightarrow 2^{X^{*}}$ be maximal monotone and $B: C \rightarrow X^{*}$ demicontinuous, bounded and pseudo-monotone. Fix $s \in X^{*}$ and assume that there exists a point $u_{s} \in D(A)$ such that $\left(B u-s, u-u_{s}\right)>0$ for all $u \in C$ with $\|u\|$ sufficiently large. Then there exists $x \in D(A)$, such that $s \in A x+B x$.

Using Lemma 1.1 and Theorem $A$, we have the following basic result for the range of the sum $A+B$.

Theorem 2.1. Let $X$ be reflexive and $X^{*}$ strictly convex. Let $C$ be a closed, convex set in $X$. Assume that $A: D(A) \subset C \rightarrow 2^{X^{*}}$ is maximal monotone and $B: C \rightarrow X^{*}$ is bounded, demicontinuous and pseudo-monotone. Let $S$ be a subset of $X^{*}$ such that: for every $s \in S$, there exist $x_{s} \in X, K(s)>0$ and $\beta=\beta_{s} \in \Gamma$ such that

$$
\left(y+B x-s, x-x_{s}\right) \geq-K(s)-\beta(\|x\|)\|x\|,
$$

for all $x \in D(A)$ with $\|x\|$ sufficiently large and all $y \in A x$. Moreover,

$$
\left(B x, x-x_{0}\right) \geq-\beta_{1}(\|x\|)\|x\|^{2} \text {, }
$$

for some $x_{0} \in D(A), \beta_{1} \in \Gamma$ and all $x \in C$ with $\|x\|$ sufficiently large. Then $S \subset \overline{R(A+B)}$ and int $S \subset$ int $R(A+B)$.

Proof. Since $X$ is a reflexive Banach space with $X^{*}$ strictly convex, the duality mapping $J$ is single-valued, bounded, demicontinuous and maximal monotone (see, for example, Zeidler [25, Proposition 32.22]). Because of this, we can show that $B+\lambda J$ is pseudo-monotone for any $\lambda>0$. For $s \in S$, let us consider the inclusion

$$
A x+B x+\frac{1}{n} J x \ni s .
$$

To show that $(2.1)$ is solvable by virtue of Theorem A, we only need to show that

$$
\left(B x+\frac{1}{n} J x-s, x-x_{0}\right)>0,
$$

for all $x \in C$ with sufficiently large $\|x\|$. To this end, we have

$$
\begin{aligned}
\left(B x+\frac{1}{n} J x-s, x-x_{0}\right)=\left(B x, x-x_{0}\right)+\frac{1}{n}\left(J x, x-x_{0}\right)-\left(s, x-x_{0}\right) \\
\quad \geq-\beta_{1}(\|x\|)\|x\|^{2}+\frac{1}{n}\|x\|^{2}-\left(\frac{1}{n}\left\|x_{0}\right\|+\|s\|\right)\|x\|-\|s\|\left\|x_{0}\right\| \\
\quad \rightarrow \infty
\end{aligned}
$$

as $\|x\| \rightarrow \infty$ because $\beta_{1}(r) \rightarrow 0$ as $\|x\| \rightarrow \infty$. Thus, we have the solvability of $(2.1)$ for any $n \in Z^{+}$.

Let $x_{n}$ be a solution of $A x+B x+(1 / n) J x \ni s$ for each $n \in Z^{+}$. We have $(1 / n) J x_{n}=-v_{n}-B x_{n}+s$, for some $v_{n} \in A x_{n}$, and, assuming that $\left\|x_{n}\right\|$ is sufficiently large,

$$
\left(\frac{1}{n} J x_{n}, x_{n}-x_{s}\right)=-\left(v_{n}+B x_{n}-s, x_{n}-x_{s}\right) \leq K(s)+\beta\left(\left\|x_{n}\right\|\right)\left\|x_{n}\right\| .
$$

This implies

$$
\frac{1}{n}\left\|x_{n}\right\|^{2} \leq \frac{1}{n}\left\|x_{s}\right\|\left\|x_{n}\right\|+\beta\left(\left\|x_{n}\right\|\right)\left\|x_{n}\right\|+K(s),
$$


which says that $(1 / n) J x_{n} \rightarrow 0$. It follows that $s \in \overline{R(A+B)}$.

Now, we are going to show int $S \subset$ int $R(A+B)$. To this end, fix $s \in$ int $S$. Then there exists $r>0$ such that for any $h \in \overline{B_{r}(0)}$ we have $s+h \in S$. Let $x_{n}$ denote a solution of $A x+B x+(1 / n) J x \ni s$ and assume that $\left\{x_{n}\right\}$ is unbounded. We may assume without loss of generality that $\left\|x_{n}\right\| \rightarrow \infty$ as $n \rightarrow \infty$. Then

$$
v_{n}+B x_{n}+\frac{1}{n} J x_{n}-(s+h)=-h, \text { for some } v_{n} \in A x_{n},
$$

and

$$
-\left(h, x_{n}-x_{s+h}\right)=\left(v_{n}+B x_{n}-(s+h), x_{n}-x_{s+h}\right)+\frac{1}{n}\left(J x_{n}, x_{n}-x_{s+h}\right) .
$$

Hence

$$
\begin{aligned}
\left(h, x_{n}\right)= & \left(h, x_{s+h}\right)-\frac{1}{n}\left(J x_{n}, x_{n}-x_{s+h}\right) \\
& -\left(v_{n}+B x_{n}-(s+h), x_{n}-x_{s+h}\right) \\
& \leq\left(h, x_{s+h}\right)+K(s+h)+\beta\left(\left\|x_{n}\right\|\right)\left\|x_{n}\right\| \\
& -\frac{1}{n}\left(\left\|x_{n}\right\|^{2}-\left\|x_{n}\right\|\left\|x_{s+h}\right\|\right) \\
& \leq\left(h, x_{s+h}\right)+K(s+h)+\beta\left(\left\|x_{n}\right\|\right)\left\|x_{n}\right\| \\
& -\frac{1}{n}\left(\left\|x_{n}\right\|^{2}-\frac{1}{2}\left(\left\|x_{n}\right\|^{2}+\left\|x_{s+h}\right\|^{2}\right)\right) \\
& \leq\left(h, x_{s+h}\right)+K(s+h)+\beta\left(\left\|x_{n}\right\|\right)\left\|x_{n}\right\|+\frac{1}{2 n}\left\|x_{s+h}\right\|^{2},
\end{aligned}
$$

for all large $n$, i.e., by Lemma 1.1 , a contradiction to the unboundedness of $\left\{x_{n}\right\}$. Thus, $\left\{x_{n}\right\}$ is bounded. Since $X$ is reflexive, $C$ is closed and convex and $\left\{x_{n}\right\} \subset C$ is bounded, we may assume that $x_{n} \rightarrow \widetilde{x}_{0} \in C$. Since $B$ is bounded, we may also assume that $B x_{n}-y_{0} \in X^{*}$.

Since $B x_{n}=-v_{n}-(1 / n) J x_{n}+s$, we have, for $x \in D(A), y \in A x$,

$$
\begin{aligned}
\left(B x_{n}, x_{n}-\tilde{x}_{0}\right)= & \left(B x_{n}, x_{n}-x\right)-\left(B x_{n}, \tilde{x}_{0}-x\right), \\
\left(B x_{n}, x_{n}-x\right) & \leq\left(B x_{n}+v_{n}-y, x_{n}-x\right) \\
& =\left(s-\frac{1}{n} J x_{n}-y, x_{n}-x\right) \\
& \rightarrow\left(s-y, \tilde{x}_{0}-x\right),
\end{aligned}
$$

and $\left(B x_{n}, \tilde{x}_{0}-x\right) \rightarrow\left(y_{0}, \tilde{x}_{0}-x\right)$, where we have used the monotonicity of the operator $A$. Thus,

$$
\begin{aligned}
\limsup _{n \rightarrow \infty}\left(B x_{n}, x_{n}-\tilde{x}_{0}\right) & \leq\left(s-y, \tilde{x}_{0}-x\right)-\left(y_{0}, \tilde{x}_{0}-x\right) \\
& =\left(s-y_{0}-y, \tilde{x}_{0}-x\right),
\end{aligned}
$$

for every $x \in D(A), y \in A x$. We also have $\inf \left\{\left(s-y_{0}-y, \tilde{x}_{0}-x\right): x \in\right.$ $D(A), y \in A x\} \leq 0$. If this is not true, then $\left(s-y_{0}-y, \tilde{x}_{0}-x\right) \geq c>0$ for every $x \in D(A), y \in A x$. Since $A$ is maximal monotone, we have $\tilde{x}_{0} \in D(A)$ and $s-y_{0} \in A \widetilde{x}_{0}$. Let $x=\tilde{x}_{0}, y=s-y_{0}$. Then $\left(s-y_{0}-y, \tilde{x}_{0}-x\right)=0$, which is a contradiction to the assumed inequality. It follows that

$$
\limsup _{n \rightarrow \infty}\left(B x_{n}, x_{n}-\tilde{x}_{0}\right) \leq 0 \text {. }
$$


Since $B$ is pseudo-monotone, hence generalized pseudo-monotone, we have $B \widetilde{x}_{0}=y_{0}$ and

$$
\left(B \tilde{x}_{0}, \tilde{x}_{0}-x\right) \leq \liminf _{n \rightarrow \infty}\left(B x_{n}, x_{n}-x\right) .
$$

Now, for any $x \in D(A), y \in A x$,

$$
\begin{aligned}
\left(s-B \tilde{x}_{0}-y, \tilde{x}_{0}-x\right) & =\left(s-y, \tilde{x}_{0}-x\right)-\left(B \tilde{x}_{0}, \tilde{x}_{0}-x\right) \\
& \geq\left(s-y, \tilde{x}_{0}-x\right)-\liminf _{n \rightarrow \infty}\left(B x_{n}, x_{n}-x\right) \\
& =\lim _{n \rightarrow \infty}\left(s-y, x_{n}-x\right)-\liminf _{n \rightarrow \infty}\left(B x_{n}, x_{n}-x\right) \\
& \geq \liminf _{n \rightarrow \infty}\left(s-y-B x_{n}, x_{n}-x\right) \\
& =\liminf _{n \rightarrow \infty}\left(v_{n}+\frac{1}{n} J x_{n}-y, x_{n}-x\right) \\
& =\liminf _{n \rightarrow \infty}\left(v_{n}-y, x_{n}-x\right)+\lim _{n \rightarrow \infty}\left(\frac{1}{n} J x_{n}, x_{n}-x\right) \\
& \geq 0 .
\end{aligned}
$$

By the fact that $A$ is a maximal monotone, we have $\tilde{x}_{0} \in D(A)$ and $s-B \widetilde{x}_{0} \in$ $A \widetilde{x}_{0}$. Thus, $s \in A \widetilde{x}_{0}+B \widetilde{x}_{0}$, which says that int $S \subset R(A+B)$. We conclude that int $S \subset$ int $R(A+B)$.

Letting $S=R(A)+R(B)$ in Theorem 2.1, we have the following result.

Theorem 2.2. Let $X$ be reflexive and $X^{*}$ strictly convex. Let $C$ be a closed, convex set in $X$. Assume $A: D(A) \subset C \rightarrow 2^{X^{*}}$ is maximal monotone and $B: C \rightarrow X^{*}$ bounded, demicontinuous and pseudo-monotone. Assume that for every $(u, v) \in D(A) \times C$ there exist $x_{u, v} \in X, K(u, v)>0$ and $\beta=\beta_{u, v} \in \Gamma$ such that

$$
\left(y+B x-y_{u}-B v, x-x_{u, v}\right) \geq-K(u, v)-\beta(\|x\|)\|x\|,
$$

for all $x \in D(A)$ with $\|x\|$ sufficiently large, all $y \in A x$ and all $y_{u} \in A u$. Moreover,

$$
\left(B x, x-x_{0}\right) \geq-\beta_{1}(\|x\|)\|x\|^{2},
$$

for some $x_{0} \in D(A), \beta_{1} \in \Gamma$ and all $x \in C$ with $\|x\|$ sufficiently large. Then $\overline{R(A+B)}=\overline{(R(A)+R(B))}$ and int $R(A+B)=\operatorname{int}(R(A)+R(B))$.

Theorem 2.2 is an extension and an improvement of Theorem 2 of Browder [7]. Browder assumed that for every $v \in D(A), u \in X$ we have

$$
(B x-B u, x-v) \geq-K(u, v)-\beta(\|x\|)\|x\| .
$$

This inequality implies both inequalities of Theorem 2.2 by letting $x_{u, v}=v$. In addition, the operator $A$ above is multi-valued and the domain of the operator $B$ is not necessarily all of $X$.

It is quite clear that by choosing different sets $S$ in Theorem 2.1 we can obtain a wide variety of results involving ranges of nonlinear operators. We provide below two theorems of this nature. The second one has several corollaries which we find to be particularly interesting.

Theorem 2.3. Let $X$ be reflexive with $X^{*}$ strictly convex. Let $C$ be a nonempty closed convex set in $X$. Assume $A: D(A) \subset C \rightarrow 2^{X^{*}}$ is maximal monotone, 
and $B: C \rightarrow X^{*}$ is bounded, demicontinuous and pseudo-monotone. Assume that for every $x_{0} \in D(A)$ there exist $K\left(x_{0}\right)>0$ and $\beta=\beta_{x_{0}} \in \Gamma$ such that

$$
\left(B x, x-x_{0}\right) \geq-K\left(x_{0}\right)-\beta(\|x\|)\|x\|,
$$

for all $x \in C$ with $\|x\|$ sufficiently large. Then $\overline{R(A)} \subset \overline{R(A+B)}$ and int $R(A)$ $\subset$ int $R(A+B)$.

Proof. Let $S=R(A)$ in Theorem 2.1. Then, for $s \in R(A)$, there exists $x_{s} \in$ $D(A)$ such that $s \in A x_{s}$. Thus, for every $x \in D(A)$ with sufficiently large $\|x\|$ and every $y \in A x$, we have

$$
\begin{aligned}
\left(y+B x-s, x-x_{s}\right) & =\left(y-s, x-x_{s}\right)+\left(B x, x-x_{s}\right) \\
& \geq-K\left(x_{s}\right)-\beta(\|x\|)\|x\| .
\end{aligned}
$$

The conclusion follows now from Theorem 2.1.

Theorem 2.4. Let $X$ be reflexive with $X^{*}$ strictly convex. Let $C$ be a closed, convex set in $X$. Assume that $A: D(A) \subset C \rightarrow 2^{X^{*}}$ is maximal monotone and $B: C \rightarrow X^{*}$ is bounded, demicontinuous and pseudo-monotone. Assume that for every $s \in X^{*}$ there exist $x_{s} \in X, K(s)>0$ and $\beta=\beta_{s} \in \Gamma$ such that

$$
\left(y+B x-s, x-x_{s}\right) \geq-K(s)-\beta(\|x\|)\|x\|,
$$

for all $x \in D(A)$ with $\|x\|$ sufficiently large and all $y \in A x$. Moreover,

$$
\left(B x, x-x_{0}\right) \geq-\beta_{1}(\|x\|)\|x\|^{2},
$$

for some $x_{0} \in D(A), \beta_{1} \in \Gamma$ and all $x \in C$ with $\|x\|$ sufficiently large. Then $R(A+B)=X^{*}$.

Proof. Let $S=X^{*}$ in Theorem 2.1 .

Corollary 2.1. Let $X$ be reflexive with $X^{*}$ strictly convex. Let $C$ be a closed, convex set in $X$. Assume that $A: D(A) \subset C \rightarrow 2^{X^{*}}$ is maximal monotone, with $0 \in D(A)$, and $B: C \rightarrow X^{*}$ is bounded, demicontinuous and pseudomonotone. Assume that for every $s \in X^{*}$ there exist $K(s)>0$ and $\beta=\beta_{s} \in \Gamma$ such that:

$$
(B x-s, x) \geq-K(s)-\beta(\|x\|)\|x\|,
$$

for all $x \in C$ with $\|x\|$ sufficiently large. Then $R(A+B)=X^{*}$.

Proof. Fix $s \in X^{*}$ and let $x_{s}=x_{0}=0, y_{0} \in A(0)$. We have

$(y+B x-s, x)=\left(y-y_{0}, x\right)+\left(B x-\left(s-y_{0}\right), x\right) \geq-K\left(s-y_{0}\right)-\beta(\|x\|)\|x\|$,

for all $x \in D(A)$ with $\|x\|$ sufficiently large, and

$$
(B x, x) \geq-K(0)-\beta(\|x\|)\|x\| \geq-\beta(\|x\|)\|x\|^{2},
$$

for all $x \in C$ with sufficiently large $\|x\|$. Thus, our conclusion follows from Theorem 2.4.

Corollary 2.2. Let $X$ be reflexive with $X^{*}$ strictly convex. Let $C$ be a closed, convex set in $X$. Assume that $A: D(A) \subset C \rightarrow 2^{X^{*}}$ is maximal monotone and strongly monotone, $B: C \rightarrow X^{*}$ is bounded, demicontinuous and pseudomonotone. Assume that there exist $x_{0} \in D(A), \beta \subseteq \Gamma$ such that

$$
\left(B x, x-x_{0}\right) \geq-\beta(\|x\|)\|x\|^{2},
$$


for all $x \in C$ with $\|x\|$ sufficiently large. Then $R(A+B)=X^{*}$.

Proof. For $s \in X^{*}, x \in D(A)$, let $y \in A x$ and $y_{0} \in A x_{0}$. We have, for $\|x\|$ sufficiently large,

$$
\begin{aligned}
\left(y+B x-s, x-x_{0}\right)= & \left(y-y_{0}, x-x_{0}\right)+\left(y_{0}, x-x_{0}\right) \\
& +\left(B x, x-x_{0}\right)-\left(s, x-x_{0}\right) \\
\geq & c\left\|x-x_{0}\right\|^{2}-\left\|y_{0}\right\|\left\|x-x_{0}\right\| \\
& -\beta(\|x\|)\|x\|^{2}-\|s\|\left\|x-x_{0}\right\| \\
& \rightarrow \infty
\end{aligned}
$$

as $\|x\| \rightarrow \infty$ because $\beta(r) \rightarrow 0$ as $r \rightarrow \infty$. Here, $c$ is the strong monotonicity constant of $A$. Thus, Corollary 2.2 follows from Theorem 2.4.

It is rather obvious that our results can be viewed as complements of the basic perturbation result, Theorem A. As Browder noticed in [7], coercivity assumptions like those of Theorem 2.4 or Corollary 2.1 are considerably weaker than those of Theorem $\mathrm{A}$, for not only could $-K(s)-\beta(\|x\|)\|x\|$ be negative, but also $-K(s)-\beta(\|x\|)\|x\|$ could approach $-\infty$ as $\|x\| \rightarrow \infty$. Corollary 2.2 says that the coercivity in the problem can be generated by the maximal monotone operator $A$ itself.

In the following results we assume that $\|B x\|$ vanishes as $\|x\| \rightarrow \infty$. The reader should notice that the various functions $\beta \in \Gamma$ are now replaced by appropriate functions $\beta=\beta(x)$. The proofs go over in this case with some trivial modifications.

Theorem 2.5. Let $X$ be a reflexive with $X^{*}$ strictly convex. Let $C$ be a nonempty, closed, convex set in $X$. Assume $A: D(A) \subset C \rightarrow 2^{X^{*}}$ is maximal monotone, and $B: C \rightarrow X^{*}$ is bounded, demicontinuous and pseudo-monotone with $\|B x\| \rightarrow 0$ as $\|x\| \rightarrow \infty$. Then $\overline{R(A)} \subset \overline{R(A+B)}$ and int $R(A) \subset$ int $R(A+B)$. Proof. For $x_{0} \in D(A), x \in C$ with $\|x\|$ sufficiently large and $y \in A x$ we have

$$
\left(B x, x-x_{0}\right) \geq-\|B x\|\left\|x-x_{0}\right\| \geq-\left\|x_{0}\right\|-\|B x\|\|x\| .
$$

By the assumption that $\|B x\| \rightarrow 0$ as $\|x\| \rightarrow \infty$ and Theorem 2.3, we have the result.

Theorem 2.6. Let $X$ be reflexive with $X^{*}$ strictly convex. Let $C$ be a closed convex set in $X$. Assume that $A: D(A) \subset C \rightarrow 2^{X^{*}}$ is maximal monotone and $B: C \rightarrow X^{*}$ is bounded, demicontinuous and pseudo-monotone with $\|B x\| \rightarrow 0$ as $\|x\| \rightarrow \infty$. Furthermore, assume that for every $s \in X^{*}$ there exist $x_{s} \in X$, $K(s)>0$ and $\beta=\beta_{s} \in \Gamma$ such that

$$
\left(y-s, x-x_{s}\right) \geq-K(s)-\beta(\|x\|)\|x\|,
$$

for all $x \in D(A)$ with $\|x\|$ sufficiently large and all $y \in A x$. Then $R(A+B)=$ $X^{*}$.

Proof. Given $s \in X^{*}$, we have

$$
\begin{aligned}
\left(y+B x-s, x-x_{s}\right) & \geq\left(y-s, x-x_{s}\right)+\left(B x, x-x_{s}\right) \\
& \geq-K(s)-\beta(\|x\|)\|x\|-\|B x\|\|x\|-\|B x\|\left\|x_{s}\right\| \\
& =-K(s)-\|B x\|\left\|x_{s}\right\|-(\beta(\|x\|)+\|B x\|)\|x\|,
\end{aligned}
$$


for all $x \in D(A)$ with sufficiently large $\|x\|$ and all $y \in A x$. Also, for every $x_{0} \in C$,

$$
\left(B x, x-x_{0}\right) \geq-\|B x\|\|x\|-\|B x\|\left\|x_{0}\right\| \geq-\|B x\|\|x\|^{2},
$$

for all $x \in C$ with sufficiently large $\|x\|$. By the assumption that $\|B x\| \rightarrow 0$ as $\|x\| \rightarrow \infty$ and Theorem 2.1, we have $X^{*} \subset R(A+B)$.

Letting $B=0$ in Theorem 2.6, we obtain the following corollary.

Corollary 2.3. Let $X$ be reflexive with $X^{*}$ strictly convex. Let $A: D(A) \subset$ $X \rightarrow 2^{X^{*}}$ be maximal monotone. Assume that for every $s \in X^{*}$ there exist $x_{s} \in X, K(s)>0$ and $\beta=\beta_{s} \in \Gamma$ such that

$$
\left(y-s, x-x_{s}\right) \geq-K(s)-\beta(\|x\|)\|x\|,
$$

for all $x \in D(A)$ with $\|x\|$ sufficiently large and all $y \in A x$. Then $R(A)=X^{*}$.

The "coercivity" inequality for $A$ in Corollary 2.3.is weaker than the usual coercivity condition for this type of results. See, for example, Theorem 2.10 of Pascali and Sburlan [23] or Corollary 32.27 of Zeidler [25].

It is clear that more results could be derived from Theorem 2.1. For example, if we let $A=0$ and $S=X^{*}$ there, we have a surjectivity result for a bounded, demicontinuous and pseudo-monotone operator $B$. We omit the details of such an investigation.

\section{COMPACT PERTURBATIONS AND COMPACT RESOLVENTS OF MAXIMAL MONOTONE OPERATORS}

In this section we assume that $A$ is maximal monotone and that either $B$ or the resolvent $(A+J)^{-1}$ is compact. We start with a useful lemma.

Lemma 3.1. Let $X$ be reflexive with $X^{*}$ strictly convex. Assume that $A: X \supset$ $D(A) \rightarrow 2^{X^{*}}$ is maximal monotone. Let $x_{0} \in D(A)$ and $J_{x_{0}}(x) \equiv$ $J\left(x-x_{0}\right)$. Then, for every $\lambda>0$, the operator $\left(A+\lambda J_{x_{0}}\right)^{-1}: X^{*} \rightarrow D(A)$ is everywhere defined, single-valued, demicontinuous and bounded. Furthermore, if $X$ is locally uniformly convex, this operator is also continuous.

Proof. The proof that $\left(A+\lambda J_{x_{0}}\right)^{-1}$ is everywhere defined, single-valued and demicontinuous is almost the same as that for the operator $(A+\lambda J)^{-1}$, which can be found, for example, in Pascali and Sburlan [23, p. 112]. Thus, we omit it.

To show that $\left(A+\lambda J_{x_{0}}\right)^{-1}$ is bounded, it suffices to show $\left\{\left(A+\lambda J_{x_{0}}\right)^{-1} y_{n}\right\}$ is bounded for any bounded sequence $\left\{y_{n}\right\} \subset X^{*}$. To this end, let $x_{n}=$ $\left(A+\lambda J_{x_{0}}\right)^{-1} y_{n}$. Then $y_{n}=v_{n}+\lambda J\left(x_{n}-x_{0}\right)$, for some $v_{n} \in A x_{n}$. Fix $v_{0} \in$ $A x_{0}$. Then we have

$$
\begin{aligned}
\lambda\left(J\left(x_{n}-x_{0}\right), x_{n}-x_{0}\right) & =\left(y_{n}, x_{n}-x_{0}\right)-\left(v_{n}, x_{n}-x_{0}\right) \\
& =\left(y_{n}, x_{n}-x_{0}\right)-\left(v_{n}-v_{0}, x_{n}-x_{0}\right)+\left(v_{0}, x_{n}-x_{0}\right),
\end{aligned}
$$

which, by the monotonicity of $A$, implies

$$
\lambda\left\|x_{n}-x_{0}\right\|^{2} \leq\left(\left\|y_{n}\right\|+\left\|v_{0}\right\|\right)\left\|x_{n}-x_{0}\right\|,
$$

i.e., the boundedness of $\left\{\left\|x_{n}\right\|\right\}$.

If $X$ is locally uniformly convex, it is well-known that $J$ is of type $\left(S_{+}\right)$. In order to show that $\left(A+\lambda J_{x_{0}}\right)^{-1}$ is continuous, let $\left\{y_{n}\right\} \in X^{*}$ with $y_{n} \rightarrow y_{0} \in X^{*}$ 
and let $u_{n}=\left(A+\lambda J_{x_{0}}\right)^{-1} y_{n}, u_{0}=\left(A+\lambda J_{x_{0}}\right)^{-1} y_{0}$. Then, for some $v_{n} \in A u_{n}$ and $v_{0} \in A u_{0}$,

$$
\begin{aligned}
\left(y_{n}-y_{0}, u_{n}-u_{0}\right) & =\left(v_{n}+\lambda J\left(u_{n}-x_{0}\right)-\left(v_{0}+\lambda J\left(u_{0}-x_{0}\right)\right), u_{n}-u_{0}\right) \\
& \geq \lambda\left(J\left(u_{n}-x_{0}\right)-J\left(u_{0}-x_{0}\right), u_{n}-x_{0}-\left(u_{0}-x_{0}\right)\right) .
\end{aligned}
$$

Since $y_{n} \rightarrow y_{0}$ and $\left(A+\lambda J_{x_{0}}\right)^{-1}$ is demicontinuous we have $u_{n} \rightarrow u_{0}$. Thus, $\left(y_{n}-y_{0}, u_{n}-u_{0}\right) \rightarrow 0$ and

$$
\limsup _{n \rightarrow \infty}\left(J\left(u_{n}-x_{0}\right), u_{n}-x_{0}-\left(u_{0}-x_{0}\right)\right) \leq 0 .
$$

Since $J$ is of type $\left(S_{+}\right), u_{n}-x_{0} \rightarrow u_{0}-x_{0}$, or $u_{n} \rightarrow u_{0}$, completing the proof of the continuity of $\left(A+\lambda J_{x_{0}}\right)^{-1}$.

The results of this section improve the maximal monotone analogues of various results of Kartsatos in [18] involving $m$-accretive operators $A$.

In the following three results we assume that $A$ has a compact resolvent, i.e., $(A+J)^{-1}$ is compact. Actually, if this is true, then the relation

$$
\left(\lambda A+\mu J_{x_{0}}\right)^{-1}(x)=\left((\lambda / \mu) A+J_{x_{0}}\right)^{-1}((1 / \mu) x)=\left(A+(\mu / \lambda) J_{x_{0}}\right)^{-1}((1 / \lambda) x),
$$

for $\lambda>0, \mu>0$ and $x_{0} \in D(A)$, shows that $\left(\lambda A+\mu J_{x_{0}}\right)^{-1}$ will be compact, for every $\lambda>0, \mu>0$, if we show this fact for $\left(A+\lambda J_{x_{0}}\right)^{-1}, \lambda>0$. An analogous statement for $(\lambda A+\mu J)^{-1}$ can be shown the same way. Let $\left\{y_{n}\right\} \subset$ $X^{*}$ be bounded and $x_{n}=\left(A+\lambda J_{x_{0}}\right)^{-1} y_{n}$. Then $y_{n} \in A x_{n}+\lambda J\left(x_{n}-x_{0}\right)=$ $A x_{n}+J x_{n}+\left(\lambda J\left(x_{n}-x_{0}\right)-J x_{n}\right)$, implying

$$
y_{n}-\left(\lambda J\left(x_{n}-x_{0}\right)-J x_{n}\right) \in(A+J) x_{n}
$$

and

$$
x_{n}=(A+J)^{-1}\left(y_{n}-\lambda J\left(x_{n}-x_{0}\right)+J x_{n}\right) .
$$

Since $\left(A+\lambda J_{x_{0}}\right)^{-1}$ is bounded, we have that $\left\{x_{n}\right\}$ and $\left\{y_{n}-\lambda\left(J\left(x_{n}-x_{0}\right)-J x_{n}\right)\right\}$ are bounded. Thus, the compactness of $(A+J)^{-1}$ implies that the sequence $\left\{x_{n}\right\}$ lies in a compact set. It follows that $\left(A+\lambda J_{x_{0}}\right)^{-1}$ is compact.

We now state and prove our first result involving compact resolvents of the operator $A$.

Theorem 3.1. Let $X$ be reflexive and locally uniformly convex with $X^{*}$ strictly convex. Let $A: X \supset D(A) \rightarrow 2^{X^{*}}$ be maximal monotone with $(A+J)^{-1}$ compact and $B: \overline{D(A)} \rightarrow X^{*}$ continuous and bounded. Furthermore, let $S \subset X^{*}$ be such that: for every $s \in S$ there exist $x_{s} \in D(A), K(s)>0$ and $\beta=\beta_{s} \in \Gamma$ such that

$$
\left(v+B x-s, x-x_{s}\right) \geq-K(s)-\beta(\|x\|)\|x\|,
$$

for all $x \in D(A)$ with $\|x\|$ sufficiently large and all $v \in A x$. Then $S \subset$ $\overline{R(A+B)}$ and int $S \subset$ int $R(A+B)$.

Proof. Given $s \in S$, there exits $x_{s} \in D(A)$ such that (3.1) holds. We consider the inclusion

$$
A x+\frac{1}{n} J\left(x-x_{s}\right)+B x \ni s,
$$

or, equivalently,

$$
y+B\left(A+\frac{1}{n} J_{x_{s}}\right)^{-1} y-s=0 .
$$


Since the operator $\left(A+\frac{1}{n} J_{x_{s}}\right)^{-1}$ is compact and $B$ is continuous, we have that $B\left(A+\frac{1}{n} J_{X_{s}}\right)^{-1}: X^{*} \rightarrow X^{*}$ is compact. By the Leray-Schauder theory, (3.2) and (3.3) are solvable if all solutions of

$$
y+t\left[B\left(A+\frac{1}{n} J_{x_{s}}\right)^{-1} y-s\right]=0
$$

are uniformly bounded with respect to $t \in[0,1]$.

Assume that this is not true. Then there exist sequences $\left\{y_{m}\right\} \subset X^{*}$ and $\left\{t_{m}\right\} \subset(0,1]$ such that

$$
y_{m}+t_{m}\left[B\left(A+\frac{1}{n} J_{x_{s}}\right)^{-1} y_{m}-s\right]=0
$$

and $\left\|y_{m}\right\| \rightarrow \infty$. Letting $x_{m}=\left(A+\frac{1}{n} J_{x_{s}}\right)^{-1} y_{m}$, we see that

$$
y_{m}=v_{m}+\frac{1}{n} J\left(x_{m}-x_{s}\right) \text {, }
$$

where $v_{m} \in A x_{m}$, and

$$
v_{m}+\frac{1}{n} J\left(x_{m}-x_{s}\right)+t_{m} B x_{m}-t_{m} s=0,
$$

implying

$$
\left\|y_{m}\right\|=\left\|t_{m}\left(B x_{m}-s\right)\right\| \leq\left\|B x_{m}\right\|+\|s\| .
$$

Since $B$ is bounded, $\left\|y_{m}\right\| \rightarrow \infty$ implies $\left\|x_{m}\right\| \rightarrow \infty$. Since

$$
\frac{1}{n} J\left(x_{m}-x_{s}\right)=-v_{m}-t_{m} B x_{m}+t_{m} s,
$$

for some $v_{m} \in A x_{m}$, we use the monotonicity of $A$ and our coercivity assumption to obtain

$$
\begin{aligned}
\frac{1}{n}\left\|x_{m}-x_{s}\right\|^{2} & =-t_{m}\left(v_{m}+B x_{m}-s, x_{m}-x_{s}\right)-\left(1-t_{m}\right)\left(v_{m}, x_{m}-x_{s}\right) \\
& \leq K(s)+\beta\left(\left\|x_{m}\right\|\right)\left\|x_{m}\right\|-\left(1-t_{m}\right)\left(v_{s}, x_{m}-x_{s}\right) \\
& \leq K(s)+\beta\left(\left\|x_{m}\right\|\right)\left\|x_{m}\right\|+\left\|v_{s}\right\|\left\|x_{m}-x_{s}\right\|,
\end{aligned}
$$

for some $v_{s} \in A x_{s}$ and all large $m$. This contradicts the fact that $\left\|x_{m}\right\| \rightarrow \infty$. It follows that (3.2) and (3.3) are solvable.

Now, let $x_{n}$ be a solution of the inclusion

$$
A x+\frac{1}{n} J\left(x-x_{s}\right)+B x \ni s .
$$

If the sequence $\left\{x_{n}\right\}$ is unbounded, we may assume that $\left\|x_{n}\right\| \rightarrow \infty$. This implies

$$
\frac{1}{n}\left(J\left(x_{n}-x_{s}\right), x_{n}-x_{s}\right)=-\left(v_{n}+B x_{n}-s, x_{n}-x_{s}\right) \leq K(s)+\beta\left(\left\|x_{n}\right\|\right)\left\|x_{n}\right\|,
$$

for all large $n$, where $v_{n} \in A x_{n}$. Thus, $(1 / n)\left\|x_{n}-x_{s}\right\| \rightarrow 0$ as $n \rightarrow \infty$. We conclude that $(1 / n)\left\|x_{n}-x_{s}\right\| \rightarrow 0$ as $n \rightarrow \infty$ in all possible cases. Consequently, $s \in \overline{R(A+B)}$, i.e., $S \subset \overline{R(A+B)}$. 
In order to show that int $S \subset$ int $R(A+B)$, we fix $s \in \operatorname{int} S$ and observe that there exists $r>0$ such that $s+h \in S$ for every $h \in \overline{B_{r}(0)}$. Let $x_{n}$ denote a solution of $A x+\frac{1}{n} J\left(x-x_{s}\right)+B x \ni s$. We have, for some $v_{n} \in A x_{n}$,

$$
v_{n}+\frac{1}{n} J\left(x_{n}-x_{s}\right)+B x_{n}-(s+h)=-h
$$

and

$$
\begin{aligned}
\left(h, x_{n}-x_{s+h}\right)= & -\left(v_{n}+B x_{n}-(s+h), x_{n}-x_{s+h}\right) \\
& -\frac{1}{n}\left(J\left(x_{n}-x_{s}\right), x_{n}-x_{s+h}\right) .
\end{aligned}
$$

Let us assume that $\left\|x_{n}\right\| \rightarrow \infty$ as $n \rightarrow \infty$. We find the estimate

$$
\begin{aligned}
\left(h, x_{n}\right)= & \left(h, x_{s+h}\right)-\left(v_{n}+B x_{n}-(s+h), x_{n}-x_{s+h}\right) \\
& -\frac{1}{n}\left(J\left(x_{n}-x_{s}\right), x_{n}-x_{s+h}\right) \\
\leq & \left(h, x_{s+h}\right)+K(s+h)+\beta\left(\left\|x_{n}\right\|\right)\left\|x_{n}\right\| \\
& -\frac{1}{n}\left(J\left(x_{n}-x_{s}\right), x_{n}-x_{s}+\left(x_{s}-x_{s+h}\right)\right) \\
\leq & \left(h, x_{s+h}\right)+K(s+h)+\beta\left(\left\|x_{n}\right\|\right)\left\|x_{n}\right\| \\
& -\frac{1}{n}\left(\left\|x_{n}-x_{s}\right\|^{2}-\frac{1}{2}\left(\left\|x_{n}-x_{s}\right\|^{2}+\left\|x_{s}-x_{s+h}\right\|^{2}\right)\right) \\
\leq & \left(h, x_{s+h}\right)+K(s+h)+\beta\left(\left\|x_{n}\right\|\right)\left\|x_{n}\right\|+\frac{1}{2}\left\|x_{s}-x_{s+h}\right\|^{2} \\
= & M(h)+\beta\left(\left\|x_{n}\right\|\right)\left\|x_{n}\right\|,
\end{aligned}
$$

for all large $n$, where $M(h)$ is a constant depending only on $h$. By Lemma $1.1,\left\{x_{n}\right\}$ is bounded, i.e., a contradiction. Thus, $\left\{x_{n}\right\}$ is bounded.

From $A x_{n}+\frac{1}{n} J\left(x_{n}-x_{s}\right)+B x_{n} \ni s$ we obtain

$$
x_{n}=\left(A+J_{x_{s}}\right)^{-1}\left[-B x_{n}+s+\left(1-\frac{1}{n}\right) J\left(x_{n}-x_{s}\right)\right] .
$$

Since $B$ is bounded, $\left\{x_{n}\right\}$ is bounded and $\left(A+J_{x_{s}}\right)^{-1}$ is compact, $\left\{x_{n}\right\}$ lies in a compact set. Thus, there exists a subsequence of $\left\{x_{n}\right\}$, which we denote by $\left\{x_{n}\right\}$ again, such that $x_{n} \rightarrow x_{0}$, for some $x_{0} \in \overline{D(A)}$. Since $B$ is continuous, $B x_{n} \rightarrow B x_{0}$. Let $v_{n} \in A x_{n}$ with $v_{n}+\frac{1}{n} J\left(x_{n}-x_{s}\right)+B x_{n}=s$. We have $v_{n} \rightarrow$ $s-B x_{0}$. Since $A$ is maximal monotone, we have $x_{0} \in D(A)$ and $s-B x_{0} \in A x_{0}$, or $s \in R(A+B)$. Consequently, int $S \subset R(A+B)$, i.e., int $S \subset \operatorname{int} R(A+B)$.

In Theorem 3.1 we assumed that (3.1) is true for some $x_{s}$ in $D(A)$. In the next result the point $x_{s}$ in (3.1) may be any point in $X$ but we have to assume that the space $X$ is uniformly convex.

Theorem 3.2. Let $X$ be uniformly convex with $X^{*}$ strictly convex. Assume that $A: X \supset D(A) \rightarrow 2^{X^{*}}$ is maximal monotone with $(A+J)^{-1}$ compact and $B: \overline{D(A)} \rightarrow X^{*}$ continuous and bounded. Furthermore, let $S \subset X^{*}$ be such that: for every $s \in S$ there exist $x_{s} \in X, K(s)>0$ and $\beta=\beta_{s} \in \Gamma$ such that

$$
\left(v+B x-s, x-x_{s}\right) \geq-K(s)-\beta(\|x\|)\|x\|,
$$


for all $x \in D(A)$ with $\|x\|$ sufficiently large and all $v \in A x$. Then $S \subset$ $\overline{R(A+B)}$ and int $S \subset$ int $R(A+B)$.

Proof. We choose a point $x_{0} \in D(A)$ and consider the inclusion

$$
A x+\frac{1}{n} J\left(x-x_{0}\right)+B x \ni s,
$$

or the equivalent equation

$$
y+B\left(A+\frac{1}{n} J_{x_{0}}\right)^{-1} y-s=0 .
$$

Let

$$
T(t, x)=t\left[B\left(t A+\frac{1}{n} J_{x_{0}}\right)^{-1} x-s\right], \quad t \in(0,1], x \in X^{*},
$$

and $T(0, x)=0, x \in X^{*}$. In order to show that $T$ is actually a homotopy of compact operators, we observe first that $T(t, \cdot)$ is compact for every $t \in$ $[0,1]$. We now fix $r>0$ and set $Q=B_{r}(0)$. We observe that the continuity of $T$ on $[0,1]$ will be uniform w.r.t $x \in Q$ if and only if it is uniform at each $t=t_{0} \in[0,1]$. In fact, this is the consequence of a simple covering argument.

For $t_{0}=0$, we have $T(0, x) \equiv 0$ and is it easy to see that the set

$$
W=\bigcup_{t \in[0,1]}\left[\left(t A+\frac{1}{n} J_{x_{0}}\right)^{-1} Q\right]
$$

is bounded. This and the boundedness of $B$ imply the boundedness of the set $B\left(t A+\frac{1}{n} J_{x_{0}}\right)^{-1} Q$ uniformly w.r.t. $t \in[0,1]$. We can now use this fact in order to see that $T(t, x)$ is continuous at $t_{0}=0$ uniformly w.r.t. to $x \in Q$.

We let $t_{0} \in(0,1], c \in(0,1]$ and

$$
K=\left\{\left(t A+\frac{1}{n} J_{x_{0}}\right)^{-1} x: x \in \bar{Q}, t \in[c, 1]\right\} .
$$

We are going to show that $\bar{K}$ is compact. To this end, let $\left\{y_{m}\right\} \subset K$ be given. We need to show that $\left\{y_{m}\right\}$ has a subsequence which converges to some point $y_{0}$ in $\bar{K}$. Since $\left\{y_{m}\right\} \subset K$, there exist $t_{m} \subset[c, 1]$ and $\left\{x_{m}\right\} \subset \bar{Q}$ such that $y_{m}=\left(t_{m} A+\frac{1}{n} J_{x_{0}}\right)^{-1} x_{m}$. Since $[c, 1]$ is compact, $Q$ is convex and $X$ is reflexive, we may assume that $t_{m} \rightarrow t_{0}$ and $x_{m} \rightarrow u_{0} \in \bar{Q}$. We also have

$$
t_{m} v_{m}+\frac{1}{n} J\left(y_{m}-x_{0}\right)=x_{m}
$$

for some $v_{m} \in A y_{m}$, or

$$
t_{0} v_{m}+\frac{1}{n} J\left(y_{m}-x_{0}\right)=x_{m}+\left(t_{0}-t_{m}\right) v_{m}
$$

and

$$
\begin{aligned}
y_{m} & =\left(t_{0} A+\frac{1}{n} J_{x_{0}}\right)^{-1}\left[x_{m}+\left(t_{0}-t_{m}\right) v_{m}\right] \\
& =\left(t_{0} A+\frac{1}{n} J_{x_{0}}\right)^{-1}\left[x_{m}+\frac{t_{0}-t_{m}}{t_{m}}\left(x_{m}-\frac{1}{n} J\left(y_{m}-x_{0}\right)\right)\right] \\
& =\left(t_{0} A+\frac{1}{n} J_{x_{0}}\right)^{-1}\left[\frac{t_{0}}{t_{m}} x_{m}-\frac{t_{0}-t_{m}}{n t_{m}} J\left(y_{m}-x_{0}\right)\right] .
\end{aligned}
$$


Since $t_{m} \geq c>0, x_{m} \in \bar{Q},\left\{y_{n}\right\}$ is bounded and $\left(t_{0} A+\frac{1}{n} J_{x_{0}}\right)^{-1}$ is compact, we have that $\left\{y_{m}\right\}$ lies in a compact set. Thus, there exists a subsequence of $\left\{y_{m}\right\}$ which converges to some $y \in X$. Hence $\bar{K}$ is compact. Since $B$ is continuous, it is uniformly continuous on $\bar{K} \subset \overline{D(A)}$.

To show that $T(t, x)$ is continuous at $t_{0} \in(0,1]$ uniformly w.r.t. $x \in Q$, we only need to show, in view of the above, that $\left(t A+\frac{1}{n} J_{x_{0}}\right)^{-1} x$ is continuous at $t_{0}$ uniformly w.r.t. $x \in Q$. To this end, let $y(t, x) \equiv\left(t A+\frac{1}{n} J_{x_{0}}\right)^{-1} x$. If $\left(t A+\frac{1}{n} J_{x_{0}}\right)^{-1}$ is not uniformly continuous at $t_{0} \in(0,1]$, then there exist $\epsilon>0$, $\left\{t_{m}\right\} \subset(0,1]$ and $\left\{x_{m}\right\} \subset Q$ such that $t_{m} \rightarrow t_{0}$ and $\left\|y\left(t_{m}, x_{m}\right)-y\left(t_{0}, x_{m}\right)\right\|>$ $\epsilon$ for all $m=1,2, \ldots$. For some $u\left(t_{m}, x_{m}\right) \in A y\left(t_{m}, x_{m}\right)$, we have

$$
x_{m}=\frac{1}{n} J\left(y\left(t_{m}, x_{m}\right)-x_{0}\right)+t_{m} u\left(t_{m}, x_{m}\right)
$$

and, for some $u\left(t_{0}, x_{m}\right) \in A y\left(t_{0}, x_{m}\right)$,

$$
x_{m}=\frac{1}{n} J\left(y\left(t_{0}, x_{m}\right)-x_{0}\right)+t_{0} u\left(t_{0}, x_{m}\right) .
$$

Moreover,

$$
\begin{aligned}
\frac{1}{n}(J( & \left.\left.y\left(t_{m}, x_{m}\right)-x_{0}\right)-J\left(y\left(t_{0}, x_{m}\right)-x_{0}\right), y\left(t_{m}, x_{m}\right)-y\left(t_{0}, x_{m}\right)\right) \\
= & -\left(t_{m} u\left(t_{m}, x_{m}\right)-t_{0} u\left(t_{0}, x_{m}\right), y\left(t_{m}, x_{m}\right)-y\left(t_{0}, x_{m}\right)\right) \\
= & -t_{m}\left(u\left(t_{m}, x_{m}\right)-u\left(t_{0}, x_{m}\right), y\left(t_{m}, x_{m}\right)-y\left(t_{0}, x_{m}\right)\right) \\
& +\left(t_{0}-t_{m}\right)\left(u\left(t_{0}, x_{m}\right), y\left(t_{m}, x_{m}\right)-y\left(t_{0}, x_{m}\right)\right) \\
\leq & \left|t_{0}-t_{m}\right|\left\|u\left(t_{0}, x_{m}\right)\right\|\left[\left\|y\left(t_{m}, x_{m}\right)\right\|+\left\|y\left(t_{0}, x_{m}\right)\right\|\right] \\
= & \left.\left|t_{0}-t_{m}\right| \frac{1}{t_{0}} \| x_{m}-\frac{1}{n} J\left(y\left(t_{0}, x_{m}\right)-x_{0}\right)\right) \|\left[\left\|y\left(t_{m}, x_{m}\right)\right\|+\left\|y\left(t_{0}, x_{m}\right)\right\|\right] .
\end{aligned}
$$

Since $Q$ is bounded, $\left\{x_{m}\right\} \subset Q,\left(t A+\frac{1}{n} J_{x_{0}}\right)^{-1}$ is uniformly bounded and $t_{m} \rightarrow t_{0}$, we have

$$
\left(J\left(y\left(t_{m}, x_{m}\right)-x_{0}\right)-J\left(y\left(t_{0}, x_{m}\right)-x_{0}\right), y\left(t_{m}, x_{m}\right)-y\left(t_{0}, x_{m}\right)\right) \rightarrow 0 .
$$

By Lemma 1.3, we have $y\left(t_{m}, x_{m}\right)-y\left(t_{0}, x_{m}\right) \rightarrow 0$, which is a contradiction. Hence $T(t, x)$ is uniformly continuous in $t$ with respect to $x \in Q$.

By the Leray-Schauder theory, in order to show that (3.4) or (3.5) is solvable, we only need to show that all possible solutions of

$$
x+t\left[B\left(t A+\frac{1}{n} J_{x_{0}}\right)^{-1} x-s\right]=0
$$

are bounded independently of $t \in[0,1]$, i.e., they all lie in a ball $Q=B_{r}(0)$, for some $r>0$. If this is not true, there exist $\left\{t_{m}\right\} \subset(0,1]$ and $\left\{y_{m}\right\} \subset X^{*}$ such that

$$
y_{m}+t_{m}\left[B\left(t_{m} A+\frac{1}{n} J_{x_{0}}\right)^{-1} y_{m}-s\right]=0
$$

and $\left\|y_{m}\right\| \rightarrow \infty$. Let $x_{m}=\left(t_{m} A+\frac{1}{n} J_{x_{0}}\right)^{-1} y_{m}$. Then $y_{m}=t_{m} v_{m}+\frac{1}{n} J\left(x_{m}-x_{0}\right)$, where $v_{m} \in A x_{m}$, and

$$
t_{m} v_{m}+\frac{1}{n} J\left(x_{m}-x_{0}\right)+t_{m} B x_{m}-t_{m} s=0,
$$


which implies

$$
\left\|y_{m}\right\|=\left\|t_{m}\left(B x_{m}-s\right)\right\| \leq\left\|B x_{m}\right\|+\|s\| \text {. }
$$

Since $B$ is bounded, $\left\|y_{m}\right\| \rightarrow \infty$ implies $\left\|x_{m}\right\| \rightarrow \infty$. From

$$
\frac{1}{n} J\left(x_{m}-x_{0}\right)=-t_{m} v_{m}-t_{m} B x_{m}+t_{m} s,
$$

for some $v_{m} \in A x_{m}$, we obtain

$$
\frac{1}{n}\left(J\left(x_{m}-x_{0}\right), x_{m}-x_{s}\right)=-t_{m}\left(v_{m}+B x_{m}-s, x_{m}-x_{s}\right)
$$

and

$$
\begin{aligned}
\frac{1}{n}\left\|x_{m}-x_{0}\right\|^{2} & =-t_{m}\left(v_{m}+B x_{m}-s, x_{m}-x_{s}\right)-\frac{1}{n}\left(J\left(x_{m}-x_{0}\right), x_{0}-x_{s}\right) \\
& \leq K(s)+\beta\left(\left\|x_{m}\right\|\right)\left\|x_{m}\right\|+\frac{1}{n}\left\|x_{m}-x_{0}\right\|\left\|x_{0}-x_{s}\right\|,
\end{aligned}
$$

for all large $m$. This contradicts the fact that $\left\|x_{m}\right\| \rightarrow \infty$. Thus, (3.4) and (3.5) are solvable. The rest of the proof follows as the proof of Theorem 3.1. It is therefore omitted.

If we replace inequality (3.1) by two inequalities, which are jointly stronger than (3.1), then we may do away with the assumption of uniform convexity of the space $X$.

Theorem 3.3. Let $X$ be reflexive and locally uniformly convex with $X^{*}$ strictly convex. Let $A: X \supset D(A) \rightarrow 2^{X^{*}}$ be maximal monotone with $(A+J)^{-1}$ compact and $B: \overline{D(A)} \rightarrow X^{*}$ continuous and bounded. Furthermore, let $S \subset X^{*}$ be such that: for every $s \in S$ there exist $p, q$ with $s=p+q, x_{s} \in X, K(p)>$ $0, K(q)>0$ and $\beta=\beta_{s} \in \Gamma$ such that

$$
\left(v-p, x-x_{s}\right) \geq-K(p)-\beta(\|x\|)\|x\|
$$

and

$$
\left(B x-q, x-x_{s}\right) \geq-K(q)-\beta(\|x\|)\|x\|,
$$

for all $x \in D(A)$ with $\|x\|$ sufficiently large and all $v \in A x$. Then $S \subset$ $\overline{R(A+B)}$ and int $S \subset$ int $R(A+B)$.

Proof. As in the proof of Theorem 3.2, we consider the inclusion

$$
A x+\frac{1}{n} J\left(x-x_{0}\right)+B x \ni s
$$

and the equivalent equation

$$
y+B\left(A+\frac{1}{n} J_{x_{0}}\right)^{-1} y-s=0 .
$$

To show (3.4) and (3.5) are solvable, we only need to show that all solutions of

$$
y+t\left(B\left(A+\frac{1}{n} J_{x_{0}}\right)^{-1} y-s\right)=0
$$

are uniformly bounded with respect to $t \in[0,1]$. 
Assume that this is not true. Then there exist sequences $\left\{y_{m}\right\} \subset X^{*}$ and $\left\{t_{m}\right\} \subset(0,1]$ such that

$$
y_{m}+t_{m}\left(B\left(A+\frac{1}{n} J_{x_{0}}\right)^{-1} y_{m}-s\right)=0
$$

and $\left\|y_{m}\right\| \rightarrow \infty$. Let $x_{m}=\left(A+\frac{1}{n} J_{x_{0}}\right)^{-1} y_{m}$. Then, as in the proof of Theorem 3.1, $\left\|x_{m}\right\| \rightarrow \infty$. From

$$
\frac{1}{n} J\left(x_{m}-x_{0}\right)=-v_{m}-t_{m} B x_{m}+t_{m} s,
$$

for some $v_{m} \in A x_{m}$, we obtain

$$
\begin{aligned}
\frac{1}{n}\left\|x_{m}-x_{0}\right\|^{2}= & \frac{1}{n}\left(J\left(x_{m}-x_{0}\right), x_{m}-x_{s}\right)+\frac{1}{n}\left(J\left(x_{m}-x_{0}\right), x_{s}-x_{0}\right) \\
= & -\left(v_{m}-t_{m} p, x_{m}-x_{s}\right)-t_{m}\left(B x_{m}-q, x_{m}-x_{s}\right) \\
& +\frac{1}{n}\left(J\left(x_{m}-x_{0}\right), x_{s}-x_{0}\right) \\
= & -\left(v_{m}-p, x_{m}-x_{s}\right)-\left(1-t_{m}\right)\left(p, x_{m}-x_{s}\right) \\
& -t_{m}\left(B x_{m}-q, x_{m}-x_{s}\right)+\frac{1}{n}\left(J\left(x_{m}-x_{0}\right), x_{s}-x_{0}\right) \\
\leq & K(p)+\beta\left(\left\|x_{m}\right\|\right)\left\|x_{m}\right\|+\|p\|\left\|x_{m}-x_{s}\right\| \\
& +K(q)+\beta\left(\left\|x_{m}\right\|\right)\left\|x_{m}\right\|+\frac{1}{n}\left\|x_{m}-x_{0}\right\|\left\|x_{s}-x_{0}\right\|,
\end{aligned}
$$

for all large $m$. This contradicts the fact that $\left\|x_{m}\right\| \rightarrow \infty$. We conclude that (3.4) and (3.5) are solvable. The rest of the proof is similar to the proof of Theorem 3.1. It is therefore omitted.

If instead of the compactness of the resolvent $(A+J)^{-1}$ we assume the compactness of the operator $B$, we can obtain a similar result. We need the following result of Nagumo [22, Theorem 7].

Theorem B. Let $G \subset X$ be open and $T:[0,1] \times \bar{G} \rightarrow X$ continuous and such that $T([0,1], \bar{G}) \subset K$, where $K$ is a compact set. Assume that $s:[0,1] \rightarrow X$ is continuous and such that $s(t) \notin(I-T(t, \cdot))(\partial G)$ for every $t \in[0,1]$. Then $d(I-T(t, \cdot), G, s(t))=$ const .

Theorem 3.4. Let $X$ be reflexive and locally uniformly convex with $X^{*}$ strictly convex. Let $A: X \supset D(A) \rightarrow 2^{X^{*}}$ be maximal monotone and $B: \overline{D(A)} \rightarrow X^{*}$ compact. Let $S \subset X^{*}$ be such that: for every $s \in S$ there exist $x_{s} \in X, K(s)>0$ and $\beta=\beta_{s} \in \Gamma$ such that

$$
\left(v+B x-s, x-x_{s}\right) \geq-K(s)-\beta(\|x\|)\|x\|,
$$

for all $x \in D(A)$ with $\|x\|$ sufficiently large and all $v \in A x$. Then $S \subset$ $\overline{R(A+B)}$. If, in addition, $A$ is of type $\left(S_{+}\right)$or $B$ is of type $(M)$, int $S \subset$ int $R(A+B)$.

Proof. For every $s \in S$ there exists $x_{s} \in X$ such that (3.1) is true. Choose a point $x_{0} \in D(A)$ and consider again the inclusion

$$
A x+\frac{1}{n} J\left(x-x_{0}\right)+B x \ni s,
$$


or the equivalent equation

$$
y+B\left(A+\frac{1}{n} J_{x_{0}}\right)^{-1} y-s=0 .
$$

The operators $B\left(t A+(1 / n) J_{x_{0}}\right)^{-1}: X^{*} \rightarrow X^{*}$ are compact for every $t \in$ $(0,1]$. Let

$$
T(t, x) \equiv t\left[B\left(t A+\frac{1}{n} J_{x_{0}}\right)^{-1} x-s\right],
$$

for $t \in(0,1], x \in X^{*}$, and $T(0, x)=0, x \in X^{*}$. In the proof of Theorem 3.2 we showed that all possible solutions of $x+T(t, x)=0$ are uniformly bounded. Let us assume that they all lie in the ball $G=B_{r}(0)$, for some $r>0$. From the proof of Lemma 3.1 we can see that the sets $\left(t A+\frac{1}{n} J_{x_{0}}\right)^{-1} \bar{G}$ are uniformly bounded. Thus, $\overline{B\left(t A+\frac{1}{n} J_{x_{0}}\right)^{-1} \bar{G}}-s=Q$ is compact. This ensures that the set $K=\{t x: t \in[0,1], x \in Q\}$ is compact since $[0,1] \times Q$ is compact and multiplication $((t, x) \rightarrow t x)$ is continuous. Obviously, $T([0,1], \bar{G}) \subset K$.

Since $T(0, x)=0$ and $Q$ is uniformly bounded, it is easy to see that $T(t, x)$ is continuous at $(0, x)$ for every $x \in G$. Given $(t, x) \in(0,1] \times G$, we shall show that $T(t, x)$ is continuous at $(t, x)$. To this end, let $y(t, x) \equiv$ $\left(t A+\frac{1}{n} J_{x_{0}}\right)^{-1} x$. We only need to show that $y(t, x)$ is continuous at $(t, x)$. This would imply that $T(t, x)$ is continuous at the same point. If $y(t, x)$ is not continuous at $(t, x)$, then there exist $\left\{t_{m}\right\} \subset(0,1]$ and $\left\{x_{m}\right\} \subset G$ such that $t_{m} \rightarrow t, x_{m} \rightarrow x$ and $\left\|y\left(t_{m}, x_{m}\right)-y(t, x)\right\|>\epsilon$ for some $\epsilon>0$. From $y(t, x)=\left(t A+\frac{1}{n} J_{x_{0}}\right)^{-1} x$, we have, for $u(t, x) \in A y(t, x)$,

$$
x=\frac{1}{n} J\left(y(t, x)-x_{0}\right)+t u(t, x)
$$

and

$$
x_{m}=\frac{1}{n} J\left(y\left(t_{m}, x_{m}\right)-x_{0}\right)+t_{m} u\left(t_{m}, x_{m}\right),
$$

where $u\left(t_{m}, x_{m}\right) \in A y\left(t_{m}, x_{m}\right)$. Using these two relations, we obtain

$$
\begin{aligned}
\frac{1}{n}( & \left.J\left(y\left(t_{m}, x_{m}\right)-x_{0}\right)-J\left(y(t, x)-x_{0}\right), y\left(t_{m}, x_{m}\right)-y(t, x)\right) \\
= & -\left(t_{m} u\left(t_{m}, x_{m}\right)-t u(t, x), y\left(t_{m}, x_{m}\right)-y(t, x)\right) \\
& +\left(x_{m}-x, y\left(t_{m}, x_{m}\right)-y(t, x)\right) \\
= & -t_{m}\left(u\left(t_{m}, x_{m}\right)-u(t, x), y\left(t_{m}, x_{m}\right)-y(t, x)\right) \\
& +\left(x_{m}-x, y\left(t_{m}, x_{m}\right)-y(t, x)\right) \\
& +\left(t-t_{m}\right)\left(u(t, x), y\left(t_{m}, x_{m}\right)-y(t, x)\right) \\
\leq & \left(\left|t_{m}-t\right|\|u(t, x)\|+\left\|x_{m}-x\right\|\right)\left\|y\left(t_{m}, x_{m}\right)-y(t, x)\right\| \\
= & \left(\left|t_{m}-t\right| \frac{1}{t}\left\|x-\frac{1}{n} J\left(y(t, x)-x_{0}\right)\right\|+\left\|x_{m}-x\right\|\right)\left\|y\left(t_{m}, x_{m}\right)-y(t, x)\right\| .
\end{aligned}
$$

Since $G$ is bounded, $\left\{x_{m}\right\} \subset G,\left(t A+\frac{1}{n} J_{x_{0}}\right)^{-1}$ is uniformly bounded, $t_{m} \rightarrow t$ and $x_{m} \rightarrow x$, we arrive at

$$
\left(J\left(y\left(t_{m}, x_{m}\right)-x_{0}\right)-J\left(y(t, x)-x_{0}\right), y\left(t_{m}, x_{m}\right)-y(t, x)\right) \rightarrow 0 .
$$

It follows that $y\left(t_{m}, x_{m}\right)-y(t, x) \rightarrow 0$ (see, for example, Pascali and Sburlan [23]), which is a contradiction. Hence $T(t, x)$ is continuous at $(t, x)$. 
By Theorem B, $d(I+T(1, \cdot), G, 0)=d(I+T(0, \cdot), G, 0)=1$, and (3.4) and (3.5) are solvable.

Let $x_{n}$ be a solution of $A x+\frac{1}{n} J\left(x-x_{0}\right)+B x \ni s$. Then

$$
\begin{aligned}
\frac{1}{n}\left\|x_{n}-x_{0}\right\|^{2} & =\frac{1}{n}\left(J\left(x_{n}-x_{0}\right), x_{n}-x_{s}\right)+\frac{1}{n}\left(J\left(x_{n}-x_{0}\right), x_{s}-x_{0}\right) \\
& =-\left(v_{n}+B x_{n}-s, x_{n}-x_{s}\right)+\frac{1}{n}\left(J\left(x_{n}-x_{0}\right), x_{s}-x_{0}\right) \\
& \leq K(s)+\beta\left(\left\|x_{n}\right\|\right)\left\|x_{n}\right\|+\frac{1}{n}\left\|x_{n}-x_{0}\right\|\left\|x_{s}-x_{0}\right\|,
\end{aligned}
$$

for all large $n$, where $v_{n} \in A x_{n}$. This implies $\frac{1}{n}\left\|x_{n}-x_{0}\right\| \rightarrow 0$ as $n \rightarrow \infty$. So, $s \in \overline{R(A+B)}$ and $S \subset \overline{R(A+B)}$.

To prove that int $S \subset \operatorname{int} R(A+B)$, let $s \in \operatorname{int} S$ and let $x_{n}$ be a solution of $A x+\frac{1}{n} J\left(x-x_{0}\right)+B x \ni s$. As in the proof of Theorem 3.1, we can show that the sequence $\left\{x_{n}\right\}$ is bounded. Since $X$ is reflexive, the set $\overline{D(A)}$ is convex and we may assume that $x_{n}-u_{0}$ for some $u_{0} \in \overline{D(A)}$. Since $B$ is compact, we may also assume that $B x_{n} \rightarrow y_{0}$, for some $y_{0} \in X^{*}$. Let $v_{n} \in A x_{n}$ be such that $v_{n}+\frac{1}{n} J\left(x_{n}-x_{0}\right)+B x_{n}=s$. Then $v_{n} \rightarrow s-y_{0}$.

If we assume that $B$ is of type $(M)$, we have $y_{0}=B u_{0}$. By the fact that $A$ is maximal monotone, we have $u_{0} \in D(A)$ and $s-B u_{0} \in A u_{0}$, or $s \in A u_{0}+B u_{0}$.

If we assume that $A$ is of type $\left(S_{+}\right)$, then $x_{n}-u_{0}$ and $v_{n} \rightarrow s-y_{0}$ imply $x_{n} \rightarrow u_{0}$. Since $B$ is continuous, $B x_{n} \rightarrow B u_{0}$. Since $A$ is maximal monotone, we have $u_{0} \in D(A)$ and $s \in A u_{0}+B u_{0}$.

If in Theorem $3.4 B$ is completely continuous, then $B$ is compact and of type $(M)$. If $A$ is strongly monotone, then $A$ is of type $\left(S_{+}\right)$. As in Section 2, by choosing the set $S$ in a more concrete way we can obtain a variety of results as a consequence of Theorems 3.1-3.4. We mention a few such situations.

Theorem 3.5. Let $X$ be reflexive and locally uniformly convex with $X^{*}$ strictly convex. Assume that $A: X \supset D(A) \rightarrow 2^{X^{*}}$ is maximal monotone with $(A+J)^{-1}$ compact and $B: \overline{D(A)} \rightarrow X^{*}$ is continuous and bounded. Furthermore, assume that for every $x_{1} \in \overline{D(A)}$ and $x_{2} \in D(A)$ there exist $K\left(x_{1}, x_{2}\right)>0$ and $\beta=$ $\beta_{x_{1}, x_{2}} \in \Gamma$ such that

$$
\left(B x-B x_{1}, x-x_{2}\right) \geq-K\left(x_{1}, x_{2}\right)-\beta(\|x\|)\|x\|
$$

for all $x \in D(A)$ with $\|x\|$ sufficiently large. Then $R(A)+R(B) \subset \overline{R(A+B)}$ and $\operatorname{int}(R(A)+R(B)) \subset \operatorname{int} R(A+B)$.

Proof. Let $S=R(A)+R(B)$ in Theorem 3.1. Then for every $s \in R(A)+R(B)$ there exist $x_{1} \in \overline{D(A)}$ and $x_{2} \in D(A)$ such that $s \in A x_{2}+B x_{1}$ or $s=v_{2}+B x_{1}$, for some $v_{2}=A x_{2}$. For $x \in D(A)$ and $v \in A x$, we let $x_{s}=x_{2}$ to obtain

$$
\begin{aligned}
(v+ & \left.B x-\left(v_{2}+B x_{1}\right), x-x_{2}\right) \\
& \geq\left(v-v_{2}, x-x_{2}\right)+\left(B x-B x_{1}, x-x_{2}\right) \\
& \geq-K\left(x_{1}, x_{2}\right)-\beta(\|x\|)\|x\|,
\end{aligned}
$$

provided that $\|x\|$ is sufficiently large. Thus, the result follows from Theorem 3.1.

Theorem 3.6. Let $X$ be reflexive and locally uniformly convex with $X^{*}$ strictly convex. Let $A: X \supset D(A) \rightarrow 2^{X^{*}}$ be maximal monotone with $(A+J)^{-1}$ 
compact and $B: \overline{D(A)} \rightarrow X^{*}$ continuous and bounded. Assume that for every $x_{0} \in D(A)$ there exist $K\left(x_{0}\right)>0$ and $\beta=\beta_{x_{0}} \in \Gamma$ such that

$$
\left(B x, x-x_{0}\right) \geq-K\left(x_{0}\right)-\beta(\|x\|)\|x\|,
$$

for all $x \in D(A)$ with $\|x\|$ sufficiently large. Then $R(A) \in \overline{R(A+B)}$ and int $R(A) \subset$ int $R(A+B)$.

Proof. Let $S=R(A)$ in Theorem 3.1. Then for every $s \in R(A)$ there exists $x_{0} \in D(A)$ such that $s \in A x_{0}$, i.e., $s=v_{0}$ for some $v_{0} \in A x_{0}$. For $x \in D(A)$ and $v \in A x$, we let $x_{s}=x_{0}$ to obtain

$$
\begin{aligned}
\left(v+B x-v_{0}, x-x_{0}\right) & \geq\left(v-v_{0}, x-x_{0}\right)+\left(B x, x-x_{0}\right) \\
& \geq-K\left(x_{0}\right)-\beta(\|x\|)\|x\|,
\end{aligned}
$$

provided that $\|x\|$ is sufficiently large. Thus, Theorem 3.6 follows from Theorem 3.1.

Theorem 3.6 extends and improves Theorem 1 of Gupta [13] and Theorem 1 of Gupta and Hess [14] (when $(A+J)^{-1}$ is compact there and not just demicontinuous and mapping bounded sets into relatively compact sets). In Gupta's result $X$ is a Hilbert space while Gupta and Hess assumed that $A$ is the sum of a monotone and a trimonotone operator. Furthermore, the "coercivity" condition on $B$ is considerably weaker in Theorem 3.6 than that in [13] or [14]. The second example in Section 5 illustrates these facts.

The following statement is an interesting corollary of Theorem 3.6.

Corollary 3.1. Let $X$ be reflexive and locally uniformly convex with $X^{*}$ strictly convex. Let $A: X \supset D(A) \rightarrow 2^{X^{*}}$ be maximal monotone with $(A+J)^{-1}$ compact and let $B: \overline{D(A)} \rightarrow X^{*}$ be continuous and bounded. Assume that $\|B x\| \rightarrow 0$ as $x \in D(A)$ with $\|x\| \rightarrow \infty$. Then $R(A) \subset \overline{R(A+B)}$ and int $R(A) \subset$ int $R(A+B)$.

Proof. For every $x_{0} \in D(A), x \in D(A)$,

$$
\begin{aligned}
\left(B x, x-x_{0}\right) & =-\left(B x, x_{0}\right)+(B x, x) \\
& \geq-\|B x\|\left\|x_{0}\right\|-\|B x\|\|x\| \\
& \geq-\left\|x_{0}\right\|-\beta(x)\|x\|,
\end{aligned}
$$

provided that $\|x\|$ is sufficiently large, with $\beta(x) \equiv\|B x\|$. Since $\beta(x) \rightarrow 0$ as $\|x\| \rightarrow \infty$, Corollary 3.1 follows easily from the proof of Theorem 3.6. The reader is directed here to the remark preceding Theorem 2.5 concerning functions of the type $\beta(x)$.

If the assumption that $(A+J)^{-1}$ is compact is replaced by the assumption of compactness of the operator $B$, then Theorem 3.4 could be used to obtain results similar to Theorem 3.5, Theorem 3.6 and Corollary 3.1.

Several results of this section can be extended to situations where the resolvent $(A+J)^{-1}$ is completely continuous and $B$ is just a demicontinuous and bounded operator defined on all of $X$. The analogous problem for $m$-accretive operators $A$ was dealt with by Kartsatos in [18].

Theorem 3.7. Let $X$ be uniformly convex and $X^{*}$ strictly convex. Assume that $A: X \supset D(A) \rightarrow 2^{X^{*}}$ is maximal monotone, with $0 \in D(A)$ and $(A+J)^{-1}$ 
completely continuous, and $B: X \rightarrow X^{*}$ is demicontinuous and bounded. Assume, further, that there exists a set $S \subset X^{*}$ such that: for every $s \in S$ there exist $x_{s} \in X, K=K(s)>0$ and $\beta=\beta_{s} \in \Gamma$ such that

$$
\left(v+B x-s, x-x_{s}\right) \geq-K(s)-\beta(\|x\|)\|x\|,
$$

for all $x \in D(A)$ with $\|x\|$ sufficiently large and all $v \in A x$. Then $S \subset$ $\overline{R(A+B)}$ and int $S \subset$ int $R(A+B)$.

Proof. Since $0 \in D(A)$, we may assume that $0 \in A(0)$. In fact, if this is not true, then we replace the operators $A, B$ by the operators $A-v$ and $B+v$, respectively, where $v$ is a fixed element in $A(0)$. We consider the inclusion

$$
A x+\frac{1}{n} J x+B x \ni s,
$$

for some fixed $s \in S$, or the equivalent equation

$$
y+\left(A+\frac{1}{n} J\right)^{-1}(B y-s)=0 .
$$

We use the homotopy mapping $T(t, x)$ defined by

$$
T(t, x)=\left(t A+\frac{1}{n} J\right)^{-1}(t(B x-s)), \quad t \in(0,1], x \in X,
$$

and $T(0, x)=0, x \in X$. It is obvious that the mapping $T(t, x)$ is compact w.r.t. $x$ for each $t \in(0,1]$, due to the fact that it is the composition of a demicontinuous and bounded operator $B$ and a completely continuous operator $(t A+(1 / n) J)^{-1}$ in a reflexive space. The fact that the complete continuity of $(A+J)^{-1}$ implies the complete continuity of $\left(\lambda A+\mu J_{x_{0}}\right)^{-1}$, for any $x_{0} \in D(A)$, follows from the following lemma.

Lemma 3.2. Let $X$ be reflexive and locally uniformly convex and let $X^{*}$ be strictly convex. Let $A: X \supset D(A) \rightarrow 2^{X^{*}}$ be maximal monotone and such that $(A+J)^{-1}$ is completely continuous. Then $(\lambda A+\mu J)^{-1}$ is completely continuous for every $\lambda, \mu>0$. Actually, $\left(\lambda A+\mu J_{x_{0}}\right)^{-1}$ is completely continuous for every $x_{0} \in D(A)$ and every $\lambda>0, \mu>0$.

Proof. From the discussion preceding Theorem 3.1 we see that it it suffices to show that $\left(A+\lambda J_{x_{0}}\right)^{-1}$ is completely continuous for every $\lambda>0$. To this end, fix $\lambda>0$ and let $\left\{y_{n}\right\}$ be a sequence in $X^{*}$ such that $y_{n} \rightarrow y_{0} \in X^{*}$. Let

$$
x_{n}=\left(A+\lambda J_{x_{0}}\right)^{-1} y_{n} .
$$

Then the compactness of $(A+J)^{-1}$ and the discussion preceding Theorem 3.1 imply that the operator $\left(A+\lambda J_{x_{0}}\right)^{-1}$ is compact. It follows that there exists a subsequence of $\left\{x_{n}\right\}$, denoted again by $\left\{x_{n}\right\}$, such that $x_{n} \rightarrow$ (some) $\tilde{x}_{0} \in$ $\overline{D(A)}$ as $n \rightarrow \infty$. Then we have

$$
x_{n}=(A+J)^{-1}\left(y_{n}-(\lambda-1) J x_{n}\right),
$$

which implies, by the complete continuity of $(A+J)^{-1}$, that

$$
\tilde{x}_{0}=(A+J)^{-1}\left(y_{0}-(\lambda-1) J \tilde{x}_{0}\right) \in D(A) .
$$

This is equivalent to

$$
\tilde{x}_{0}=(A+\lambda J)^{-1} y_{0}
$$


Since we could have started with any subsequence of $\left\{x_{n}\right\}$, instead of $\left\{x_{n}\right\}$ itself, we have actually shown the following: every subsequence of $\left\{x_{n}\right\}$ contains a further subsequence which converges to the point $(A+\lambda J)^{-1} y_{0}$. Thus, $\left\{x_{n}\right\}$ itself converges strongly to this point, and we have the complete continuity of $(A+\lambda J)^{-1}$.

Proof of Theorem 3.7 Continued. It follows that $T(t, \cdot)$ is compact for every $t \in[0,1]$. In order to show that $T(t, x)$ is continuous in $t$ uniformly w.r.t. $x$ lying in any closed ball $\bar{Q}$ with $Q=B_{r}(0)$, we show first that $T(t, x)$ is continuous in $t$ at $t=0$ uniformly w.r.t. $x \in \bar{Q}$. To this end, we observe first that, as in the case of the set $W$ in the proof of Theorem 3.2, the set

$$
\bigcup_{t \in[0,1]}\left[\left(t A+\frac{1}{n} J\right)^{-1}(t(B \bar{Q}-s))\right]
$$

is bounded. To see this, let $\left\{t_{m}\right\} \subset[0,1],\left\{x_{m}\right\} \subset \bar{Q}$. and

$$
u_{m}=\left(t_{m} A+\frac{1}{n} J\right)^{-1}\left(t_{m}\left(B x_{m}-s\right)\right) \text {. }
$$

Then, for some $v_{m} \in A u_{m}$,

$$
t_{m} v_{m}+\frac{1}{n} J u_{m}=t_{m}\left(B x_{m}-s\right)
$$

which implies

$$
0 \leq \frac{1}{n}\left\|u_{m}\right\|^{2} \leq t_{m}\left(v_{m}, u_{m}\right)+\frac{1}{n}\left\|u_{m}\right\|^{2} \leq t_{m}\left(\left\|B x_{m}\right\|+\|s\|\right)\left\|u_{m}\right\| .
$$

This implies in turn the boundedness of $\left\{u_{m}\right\}$.

In order to show that $T(t, x)$ is continuous in $t$ at $t=0$ uniformly w.r.t. $x \in \bar{Q}$, let us assume that this is not true. Then there exists a sequence $\left\{t_{m}\right\} \subset$ $(0,1]$ such that $t_{m} \rightarrow 0^{+}$and a sequence $\left\{x_{m}\right\} \subset \bar{Q}$ such that the sequence $\left\{u_{m}\right\}$, defined as above, satisfies $\left\|u_{m}\right\|>\epsilon, m \in Z^{+}$, where $\epsilon$ is a positive constant. Since (3.11) still holds in this case, we get the contradiction that $u_{m} \rightarrow$ 0 as $m \rightarrow \infty$.

Now, for the continuity of $T(t, x)$ in $t \in(0,1]$, uniformly w.r.t. $x \in \bar{Q}$, we observe first that there exists a closed ball $\overline{B_{\mu}(0)}$ such that

$$
\bigcup_{t \in(0,1]}[t(B \bar{Q}-s)] \subset \overline{B_{\mu}(0)} .
$$

A careful examination of the proof of Theorem 3.2 shows that the operator $y(t, x)$ in that proof (with $x_{0}=0$ ) has this uniform continuity property with the set $Q$ there replaced by the ball $\overline{B_{\mu}(0)}$. Now, fix $t_{0} \in(\dot{\mathrm{i}}, 1]$ and let $\left\{t_{m}\right\} \subset$ $(0,1]$ be such that $t_{m} \rightarrow t_{0}$. We have, for $x \in \bar{Q}$,

$$
\begin{aligned}
\|\left(t_{m} A+\right. & \left.\frac{1}{n} J\right)^{-1}\left(t_{m}(B x-s)\right)-\left(t_{0} A+\frac{1}{n} J\right)^{-1}\left(t_{0}(B x-s)\right) \| \\
\leq & \left\|\left(t_{m} A+\frac{1}{n} J\right)^{-1}\left(t_{m}(B x-s)\right)-\left(t_{0} A+\frac{1}{n} J\right)^{-1}\left(t_{m}(B x-s)\right)\right\| \\
& +\left\|\left(t_{0} A+\frac{1}{n} J\right)^{-1}\left(t_{m}(B x-s)\right)-\left(t_{0} A+\frac{1}{n} J\right)^{-1}\left(t_{0}(B x-s)\right)\right\| \\
\equiv & I_{1}+I_{2} .
\end{aligned}
$$


The term $I_{1}$ can be handled by the above discussion on the continuity of the operator $y(t, x)$. For the term $I_{2}$, let

$$
u_{m}(x)=t_{m}(B x-s), v_{m}(x)=\left(t_{0} A+\frac{1}{n} J\right)^{-1} u_{m}(x)
$$

and

$$
u_{0}(x)=t_{0}(B x-s), v_{0}(x)=\left(t_{0} A+\frac{1}{n} J\right)^{-1} u_{0}(x)
$$

We have

$$
\left(t_{0} A v_{m}(x)+\frac{1}{n} J v_{m}(x)\right)-\left(t_{0} A v_{0}(x)+\frac{1}{n} J v_{0}(x)\right) \ni u_{m}(x)-u_{0}(x),
$$

which implies, by the monotonicity of $A$,

$$
\left\|v_{m}(x)-v_{0}(x)\right\| \leq n\left\|u_{m}(x)-u_{0}(x)\right\| \leq L n\left|t-t_{0}\right|,
$$

where

$$
L=\sup _{x \in \bar{Q}}\{\|B x-s\|\}<+\infty .
$$

This completes the proof of the continuity of $T(t, x)$.

It follows that the Leray-Schauder degree $d(I+T(t, \cdot), Q, 0)$ is well-defined for any ball $Q=B_{r}(0)$, provided that the equation $x+T(t, x)=0$ has no solution $x \in \partial B_{r}(0)$.

In order to show that there is $r>0$ such that

$$
d(I+T(t, \cdot), Q, 0)=d(I+T(0, \cdot), Q, 0)=1,
$$

for every $t \in[0,1]$, we must show that all possible solutions $x_{t}$ of the equation $x_{t}+T\left(t, x_{t}\right)=0$ are uniformly bounded. However, since

$$
t A x_{t}+t B x_{t}+\frac{1}{n} J x_{t}-t s \ni 0
$$

this assertion follows exactly as the last part of the proof of Theorem 3.2. It is therefore omitted. It follows that (3.10) is solvable. If we let $x_{n}$ denote a solution of (3.10), we see easily, exactly as in the proof of Theorem 3.2, that $(1 / n) x_{n} \rightarrow 0$, i.e., $s \in \overline{R(A+B)}$.

To show our second assertion, let $s, r, h$ be as in the proof of Theorem 3.1 and let $x_{n}$ denote a solution of (3.10). Then we may repeat the proof of Theorem 3.1, with $x_{s}$ replaced by 0 , to obtain that $\left\{x_{n}\right\}$ is a bounded sequence. Then the relation

$$
x_{n}=(A+J)^{-1}\left[-B x_{n}+s+\left(1-\frac{1}{n}\right) J x_{n}\right],
$$

the boundedness of $B$ and the compactness of $(A+J)^{-1}$ imply that $\left\{x_{n}\right\}$ lies in a compact set. Thus, we may assume that $x_{n} \rightarrow x_{0} \in \overline{D(A)}$. From the demicontinuity $B$, we obtain that, for some $v_{n} \in A x_{n}$, we have $v_{n} \rightarrow$ $s-B x_{0}$. Since $A$ is maximal monotone, it is demiclosed. Thus, $x_{0} \in D(A)$ and $s-B x_{0} \in A x_{0}$. This completes the proof.

\section{Perturbations of $m$-ACCretive operators}

In this section we study the ranges of operators $A+B$ with $m$-accretive operators $A$. We give three theorems in this direction. In view of the results of 
the preceding section, the reader will have no difficulty in obtaining variations to these theorems as well as several corollaries other than the ones included herein. Various results of Calvert and Gupta [8] and Kartsatos [18] are improved and/or extended.

Theorem 4.1. Let $A: X \supset D(A) \rightarrow 2^{X}$ be m-accretive with $(A+I)^{-1}$ compact, and let $B: \overline{D(A)} \rightarrow X$ be continuous and bounded. Let $S \subset X$ be such that: for every $s \in S$ there exist $K(s)>0$ and $\beta=\beta_{s} \in \Gamma$ such that

$$
(v+B x-s, j) \geq-K(s)-\beta(\|x\|)\|x\|,
$$

for all $x \in D(A)$ with $\|x\|$ sufficiently large, all $v \in A x$ and some $j=j_{x, s} \in$ $J x$. Then $S \subset \overline{R(A+B)}$ and int $S \subset R(A+B)$.

Proof. For $s \in S$, we consider the inclusion

$$
A x+B x+\frac{1}{n} x \ni s
$$

and the equivalent equation

$$
x+B\left(A+\frac{1}{n} I\right)^{-1} x-s=0 .
$$

By the assumptions that $(A+I)^{-1}$ is compact and $B$ is continuous, the operator $B(\lambda A+\mu I)^{-1}: X \rightarrow X$ is compact for every $\lambda>0, \mu>0$ by the resolvent identity. Let

$$
T(t, x) \equiv t\left[B\left(t A+\frac{1}{n} I\right)^{-1} x-s\right], \quad \text { for } t \in(0,1], x \in X,
$$

and $T(0, x)=0, x \in X$. Also, let $Q \equiv B_{r}(0) \subset X$, for $r>0$.

We are going to show first that $T(t, x)$ is uniformly continuous in $t \in[0,1]$ with respect to $x \in Q$, for any $r>0$.

Since the set $\left(t A+\frac{1}{n} I\right)^{-1} Q$ is uniformly bounded and $B$ is bounded, we have that the set $B\left(t A+\frac{1}{n} I\right)^{-1} Q-s$ is uniformly bounded. Using this fact and the fact that $T(0, x) \equiv 0$, it is easy to see that $T(t, x)$ is continuous at $t_{0}=0$ uniformly w.r.t. $x \in Q$.

We now show that $T(t, x)$ is continuous at $t_{0} \in(0,1]$ uniformly w.r.t. $x \in Q$. To this end, we show first that $\bar{K}$ is compact, where

$$
K \equiv\left\{(t A+(1 / n) I)^{-1} x: x \in \bar{Q}, t \in[c, 1]\right\},
$$

for any constant $c>0$. In order to see this, let $\left\{y_{m}\right\} \subset K$. We need to show $\left\{y_{m}\right\}$ has a convergent subsequence. Let $y_{m} \equiv\left(t_{m} A+\frac{1}{n} I\right)^{-1} x_{m}$, for some $\left\{x_{m}\right\} \subset \bar{Q}$ and $\left\{t_{m}\right\} \subset[c, 1]$. For some $v_{m} \in A y_{m}$,

$$
t_{m} v_{m}+\frac{1}{n} y_{m}=x_{m}
$$

or

$$
t_{0} v_{m}+\frac{1}{n} y_{m}=x_{m}-\left(t_{m}-t_{0}\right) v_{m}
$$


for a fixed $t_{0} \in[c, 1]$, or

$$
\begin{aligned}
y_{m} & =\left(t_{0} A+\frac{1}{n} I\right)^{-1}\left(x_{m}-\left(t_{m}-t_{0}\right) v_{m}\right) \\
& =\left(t_{0} A+\frac{1}{n} I\right)^{-1}\left[x_{m}-\left(t_{m}-t_{0}\right) \frac{1}{t_{m}}\left(x_{m}-\frac{1}{n} y_{m}\right)\right] \\
& =\left(t_{0} A+\frac{1}{n} I\right)^{-1}\left[\frac{t_{0}}{t_{m}} x_{m}+\frac{t_{m}-t_{0}}{n t_{m}} y_{m}\right] .
\end{aligned}
$$

Since $\left\{t_{m}\right\} \subset[c, 1],\left\{x_{m}\right\} \subset \bar{Q}$ and $\left(t A+\frac{1}{n} I\right)^{-1}$ is uniformly bounded, we have that the expression in the last display brackets above is bounded. The fact that $\left(t_{0} A+\frac{1}{n} I\right)^{-1}$ is compact implies that $\left\{y_{m}\right\}$ lies in a compact set. Thus, there exists a subsequence of $\left\{y_{m}\right\}$, which we denote again by $\left\{y_{m}\right\}$, such that $y_{m} \rightarrow y$, for some $y \in X$. It follows that $\bar{K}$ is compact.

Since $B$ is continuous, $B$ is uniformly continuous on $\bar{K}$ and, in particular, on $K$. To show that $T(t, x)$ is uniformly continuous at $t_{0} \neq 0$, we only need to show $y(t, x)=\left(t A+\frac{1}{n} I\right)^{-1} x$ is continuous at $t_{0}$ uniformly w.r.t. $x \in Q$ and then apply a simple covering argument. This part of the proof can be found in Kartsatos [17, Proof of Theorem 3]. It is therefore omitted.

Before we apply the Leray-Schauder theory on a specific ball $Q$, we are going to show that all solutions of

$$
x+t\left[B\left(t A+\frac{1}{n} I\right)^{-1} x-s\right]=0,
$$

for $t \in(0,1]$, lie in a fixed ball $B_{r}(0)$, for some $r>0$. If this is not true, there exist sequences $\left\{x_{m}\right\} \subset X,\left\{t_{m}\right\} \subset(0,1]$ such that

$$
x_{m}+t_{m}\left[B\left(t_{m} A+\frac{1}{n} I\right)^{-1} x_{m}-s\right]=0
$$

and $\left\|x_{m}\right\| \rightarrow \infty$. Letting $y_{m}=\left(t_{m} A+\frac{1}{n} I\right)^{-1} x_{m}$, we have $t_{m} v_{m}+\frac{1}{n} y_{m}=x_{m}$, for some $v_{m} \in A y_{m}$, and

$$
t_{m} v_{m}+\frac{1}{n} y_{m}+t_{m} B y_{m}-t_{m} s=0
$$

which implies

$$
\left\|x_{m}\right\|=\left\|-t_{m} B y_{m}+t_{m} s\right\| \leq\left\|B y_{m}\right\|+\|s\| .
$$

By the fact that $B$ is bounded and $\left\|x_{m}\right\| \rightarrow \infty$, we have $\left\|y_{m}\right\| \rightarrow \infty$ and

$$
\frac{1}{n} y_{m}=-t_{m}\left(v_{m}+B y_{m}-s\right)
$$

which yields, for an appropriate functional $j=j_{m} \in J y_{m}$,

$$
\begin{aligned}
\frac{1}{n}\left\|y_{m}\right\|^{2} & =-t_{m}\left(v_{m}+B y_{m}-s, j\right) \\
& \leq K(s)+\beta\left(\left\|y_{m}\right\|\right)\left\|y_{m}\right\|,
\end{aligned}
$$

for all large $m$, i.e., a contradiction to the unboundedness of $\left\{y_{m}\right\}$. Thus, all solutions of $x+T(t, x)=0$ are in $Q \equiv B_{r}(0)$, for some $r>0$.

By the Leray-Schauder degree theory, we have

$$
d(I+T(1, \cdot), Q, 0)=d(I+T(0, \cdot), Q, 0)=1 .
$$


It follows that (4.2) and (4.1) are solvable for each $n$.

Let $x_{n}$ be a solution of $A x+B x+\frac{1}{n} x \ni s$, for each $n \in Z^{+}$. Then

$$
\frac{1}{n} x_{n}=-\left(v_{n}+B x_{n}-s\right)
$$

for some $v_{n} \in A x_{n}$, and, for some $j=j_{n} \in J x_{n}$,

$$
\frac{1}{n}\left\|x_{n}\right\|^{2}=-\left(v_{n}+B x_{n}-s, j\right) \leq K(s)+\beta\left(\left\|x_{n}\right\|\right)\left\|x_{n}\right\| .
$$

This implies $(1 / n) x_{n} \rightarrow 0$ as $n \rightarrow \infty$. Thus, $s \in \overline{R(A+B)}$.

To show that int $S \subset R(A+B)$, fix $s \in$ int $S$. Then there exists $r>0$ such that $s+h \in S$, for any $h \in X$ with $\|h\| \leq r$. Let $x_{n}$ denote a solution of $A x+B x+\frac{1}{n} x \ni s$ and assume that $\left\{x_{n}\right\}$ is unbounded. We have

$$
v_{n}+B x_{n}+\frac{1}{n} x_{n}-(s+h)=-h_{*}
$$

which implies, for an appropriate functional $j=j_{n} \in J x_{n}$,

$$
(h, j)=-\left(v_{n}+B x_{n}-(s+h), j\right)-\frac{1}{n}\left(x_{n}, j\right) .
$$

Thus,

$$
(h, j) \leq-\left(v_{n}+B x_{n}-(s+h), j\right) \leq K(s+h)-\beta\left(\left\|x_{n}\right\|\right)\left\|x_{n}\right\|,
$$

for large $n$, from which we obtain, by Lemma 1.2, that $\left\{x_{n}\right\}$ is bounded, i.e., a contradiction. Thus, $\left\{x_{n}\right\}$ is bounded. We also have

or

$$
v_{n}+B x_{n}+\frac{1}{n} x_{n}=s
$$

or

$$
v_{n}+x_{n}=s-B x_{n}+\left(1-\frac{1}{n}\right) x_{n},
$$

$$
x_{n}=(A+I)^{-1}\left[s-B x_{n}+\left(1-\frac{1}{n} x_{n}\right)\right] \text {. }
$$

Since $\left\{x_{n}\right\}$ is bounded, $B$ is bounded and $(A+I)^{-1}$ is compact, we have that $\left\{x_{n}\right\}$ lies in a compact set. We may thus assume that $x_{n} \rightarrow x_{0} \in \overline{D(A)}$. Since $B$ is continuous, we have $B x_{n} \rightarrow B x_{0}$ and $v_{n} \rightarrow s-B x_{0}$. By the fact that $A$ is $m$-accretive, and thus closed, we have $x_{0} \in D(A)$ and $s-B x_{0} \in A x_{0}$ or int $S \in$ int $R(A+B)$.

The above proof illuminates further the proof of Theorem 5 in [18]. For special sets $S$ in the above theorem, we have the following important corollaries.

Corollary 4.1. Let $A: X \supset D(A) \rightarrow 2^{X}$ be m-accretive with $(A+I)^{-1}$ compact, and let $B: \overline{D(A)} \rightarrow X$ be continuous and bounded. Assume that for every $p \in B_{r}(0)$, for some $r>0$, there exist $K(p)>0$ and $\beta=\beta_{p} \in \Gamma$ such that

$$
(v+B x-p, j) \geq-K(p)-\beta(\|x\|)\|x\|
$$

for all $x \in D(A)$ with $\|x\|$ sufficiently large, all $v \in A x$ and some $j=j_{x, p} \in$ $J x$. Then $B_{r}(0) \subset R(A+B)$.

Proof. Just take $S=B_{r}(0)$ in Theorem 4.1 . 
Corollary 4.2. Let $A: X \supset D(A) \rightarrow 2^{X}$ be m-accretive with $(A+I)^{-1}$ compact, and let $B: \overline{D(A)} \rightarrow X$ be continuous and bounded. Assume that for some $p \in X$ there exist $K>0$ and $\beta \in \Gamma$ such that

$$
(v+B x-p, j) \geq-K-\beta(\|x\|)\|x\|
$$

for all $x \in D(A)$ with $\|x\|$ sufficiently large, all $v \in A x$ and some $j=j_{x} \in J x$. Then $p \in \overline{R(A+B) \text {. }}$

Proof. Just take $S=\{p\}$ in Theorem 4.1.

We should note here that the boundary condition (4.4) is considerably weaker than the condition

$$
(v+B x-p, j) \geq 0,
$$

which was studied by Kartsatos in [17, Theorem 5]. However, the conclusion $p \in \overline{R(A+B)}$ in Theorem 4.1 was derived from the fact that the sequence $\left\{(1 / n) x_{n}\right\}$ converges to zero as $n \rightarrow \infty$. This property does not insure that the sequence $\left\{x_{n}\right\}$ is bounded, which is true in the proof of Theorem 5 in [17]. Due to this fact, we need new feasible conditions on $A, B$, other that (4.5), which will force the point $p$ to lie in the set $R(A+B)$. This was the conclusion of Theorem 5 in [17]. On the other hand, under (4.3), which is nothing else than (4.4) for every $p \in B_{r}(0)$, we can insure that the entire ball $B_{r}(0)$ lies in $R(A+B)$, which is a considerable improvement over a possible analogous statement for Theorem 5 in [17].

The problem thus arises as to what additional assumptions should be placed upon the operators $A, B$ so that the solutions $x_{n}$ of the problem 4.1 with $s=p$, with the property $(1 / n) x_{n} \rightarrow 0$ as $n \rightarrow \infty$, are actually uniformly bounded for a fixed point $p \in X$. Analogous remarks hold for Theorem 4.2 below as well as various results in the previous section.

Theorem 4.2. Let $A: X \supset D(A) \rightarrow 2^{X}$ be m-accretive and let $B: \overline{D(A)} \rightarrow X$ be compact. Let $S \subset X$ be such that: for every $s \in S$ there exist $K(s)>0$ and $\beta=\beta_{s} \in \Gamma$ such that

$$
(v+B x-s, j) \geq-K(s)-\beta(\|x\|)\|x\|,
$$

for all $x \in D(A)$ with $\|x\|$ sufficiently large, some $j=j_{x, s} \in J x$ and all $v \in A x$. Then $S \subset \overline{R(A+B)}$. If, moreover, $X$ is uniformly convex and $B$ is completely continuous, then int $S \subset$ int $R(A+B)$.

Proof. For $s \in S$, we consider again the inclusion

$$
A x+B x+\frac{1}{n} x \ni s
$$

or the equivalent equation

$$
x+B\left(A+\frac{1}{n} I\right)^{-1} x-s=0 .
$$

Letting $T(t, x) \equiv t\left[B\left(t A+\frac{1}{n} I\right)^{-1}-s\right]$, we can use Theorem B to show the solvability of (4.1). For this proof, we refer the reader to Theorem 3 of Kartsatos [17] and Theorem 6 of Kartsatos [19].

Let $x_{n}$ be a solution of (4.1). We have

$$
A x_{n}+B x_{n}+\frac{1}{n} x_{n} \ni s
$$


and, for an appropriate $j=j_{n} \in J x_{n}$,

$$
\frac{1}{n}\left\|x_{n}\right\|^{2}=-\left(v_{n}+B x_{n}-s, j\right) \leq K(s)+\beta\left(\left\|x_{n}\right\|\right)\left\|x_{n}\right\|,
$$

which implies $\frac{1}{n} x_{n} \rightarrow$ as $n \rightarrow \infty$. Consequently, $s \in \overline{R(A+B)}$.

To show the second part of the theorem, let $s \in$ int $S$ and let $x_{n}$ be again a solution of $A x+B x+\frac{1}{n} x \ni s$, for $n \in Z^{+}$. As in the proof of Theorem 4.1, we have that $\left\{x_{n}\right\}$ is bounded. Then, by Lemma 1 of Guan and Kartsatos [10], we can easily see that $s \in R(A+B)$. It follows that int $S \subset$ int $R(A+B)$.

Naturally, corollaries analogous to 4.1 and 4.2 hold for Theorem 4.2 as well. The next result is an " $m$-accretive" analogue of Theorem 3.7.

Theorem 4.3. Let $X^{*}$ be uniformly convex. Assume that $T: X \supset D(T) \rightarrow 2^{X}$ is $m$-accretive with $0 \in D(A)$ and such that $(A+I)^{-1}$ is completely continuous. Assume that $B: X \rightarrow X$ is demicontinuous and bounded. Let $S \subset X$ be such that: for every $s \in S$ there exist $K(s)>0$ and $\beta=\beta_{s} \in \Gamma$ such that

$$
(v+B x-s, j) \geq-K(s)-\beta(\|x\|)\|x\|,
$$

for all $x \in D(A)$ with $\|x\|$ sufficiently large, all $v \in A x$ and some $j=j_{x, s} \in$ $J x$. Then $S \subset \overline{R(A+B)}$ and int $S \subset R(A+B)$.

Proof. The proof is modelled after that of Theorem 3.7. As in the proof of that theorem, we may (and do) assume that $0 \in A(0)$. We consider the inclusion (4.1), or equivalently, equation (4.2). We also consider the homotopy equation $x+T(t, x)=0$, where

$$
T(t, x)=\left(t A+\frac{1}{n} I\right)^{-1}(t(B x-s)), \quad t \in[0,1], x \in X,
$$

and $T(0, x)=0, x \in X$. It is easy to see that $T(t, x)$ is a compact operator for each $t \in[0,1]$. In fact, this follows from the reflexivity of $X$ and the complete continuity of $(A+I)^{-1}$. The complete continuity of $(\lambda A+\mu I)^{-1}$, for every $\lambda>0, \mu>0$, has been shown in the authors' paper [12].

Letting $Q$ be a bounded set in $X$, we observe that since $0 \in A(0)$, we have

$$
\left\|\left(t A+\frac{1}{n} I\right)^{-1} x\right\|=\left\|(n t A+I)^{-1}(n x)\right\| \leq n\|x\|,
$$

for every $x \in X$, which implies

$$
\|T(t, x)\| \leq n t\|B x-s\| \leq n t L,
$$

where $L$ is an upper bound for $\|B x-s\|$ on $\bar{Q}$. It follows that the operator $T(t, x)$ is continuous in $t$ at $t=0$ uniformly w.r.t. $x \in \bar{Q}$. In order to show the same property of $T(t, x)$ at $t=t_{0}>0$, we let $\left\{t_{m}\right\} \subset[0,1]$ be such that $t_{m} \rightarrow t_{0}$. Then, letting $u_{m}(x), v_{m}(x), u_{0}(x), v_{0}(x)$ be as in the proof of Theorem 3.7 (with $I$ in place of $J$ ), we obtain from an inequality like (3.11) (again, with $I$ in place of $J$ ) that $(3.12)$ holds. It remains to show that $I_{1}$ in 
(3.11) is continuous at $t=t_{0}$ uniformly w.r.t. $x \in \bar{Q}$. We have

$$
\begin{aligned}
\|\left(t_{m} A+\frac{1}{n} I\right)^{-1}\left(t_{m}(B x-s)\right) & -\left(t_{0} A+\frac{1}{n} I\right)^{-1}\left(t_{m}(B x-s)\right) \| \\
& \leq \frac{2\left|t_{m}-t_{0}\right|}{t_{0}}\left\|n t_{m}(B x-s)\right\| \\
& \leq \frac{2 n\left|t_{m}-t_{0}\right|}{t_{0}}\|B x-s\| \\
& \leq \frac{2 n L\left|t_{m}-t_{0}\right|}{t_{0}} .
\end{aligned}
$$

Here, we have used estimates from the proof of Theorem 3 of [17]. It follows that the Leray-Schauder degree $d(I+T(t, \cdot), Q, 0)$ is well-defined for any ball $Q=B_{r}(0)$, provided that the equation $x+T(t, x)$ has no solution on $\partial B_{r}(0)$.

The proof now follows the steps of the proof of Theorem 4.1. We only remark here that the operator $A$ is demiclosed, being $m$-accretive in a space $X$ with $X^{*}$ uniformly convex.

\section{DisCUSSION-EXAMPLES}

Pseudo-monotone operators can be found in Zeidler [25, p. 590], where the following boundary value problem is considered.

$$
\begin{gathered}
-\sum_{i=1}^{N} D_{i}\left(F_{i}(D u)\right)+g(u)=f \text { on } G, \\
u=0 \text { on } \partial G,
\end{gathered}
$$

where $G$ is a bounded region in $R^{N}$ with a sufficiently smooth boundary. Here, $N \geq 1$ and $D u=\left(D_{1} u, D_{2} u, \ldots, D_{N} u\right)$. For $x=\left(\xi_{1}, \xi_{2}, \ldots, \xi_{N}\right) \in R^{N}$, we set $D_{i}=\partial / \partial \xi_{i}$. Conditions are imposed in [25], on the functions $F_{i}, g$, so that the principal part of the above problem defines, via an appropriate functional $a_{1}(\cdot, \cdot)$, a continuous maximal monotone operator $T_{1}: W_{0}^{1, p}(G) \rightarrow$ $W^{-1, q}(G), p \in[2, \infty)$, while the Nemytskii operator defines, via a functional $a_{2}(\cdot, \cdot)$, a completely continuous operator $T_{2}: W_{0}^{1, p}(G) \rightarrow W^{-1, q}(G)$. The operator $T_{2}$ is thus pseudo-monotone. Here, we should take $X=W_{0}^{1, p}$. The functionals $a_{1}, a_{2}$ are given by

$$
a_{1}(u, v)=\int_{G} \sum_{i=1}^{N} F_{i}(D u(x)) D_{i} v(x) d x\left(=\left(T_{1} u, v\right)\right)
$$

and

$$
a_{2}(u, v)=\int_{G} g(u(x)) v(x) d x\left(=\left(T_{2} u, v\right)\right),
$$

respectively. In order to apply Theorem 2.3 , we need a condition on the operator $T_{2}$, which reads as follows: for each $u_{0} \in X$ there exist $K\left(u_{0}\right)>0$ and $\beta=\beta_{u_{0}} \in \Gamma$ such that

$$
\left(T_{2} u, u-u_{0}\right)=\int_{G} g(u(x))\left(u(x)-u_{0}(x)\right) d x \geq-K\left(u_{0}\right)-\beta(\|u\|)\|u\|,
$$

for all $u \in X$ with $\|u\|$ sufficiently large. The conclusion is then that $R\left(T_{1}\right) \subset$ $\overline{R\left(T_{1}+T_{2}\right)}$ and int $R\left(T_{1}\right) \subset R\left(T_{1}+T_{2}\right)$. The reader should note that we are 
not assuming here that $T_{1}$ satisfies a coercivity condition (like Condition $\left(\mathrm{H}_{4}\right)$ of Zeidler [25, p. 590]).

We may also obtain important examples of continuous maximal monotone operators $T: X \equiv W^{1, p}(G) \rightarrow X^{*}$ defined by functionals of the type

$$
\begin{aligned}
a(u, v) \equiv & \sum_{k=1}^{N} \int_{G} \alpha_{k}(x)\left|\frac{\partial u}{\partial x_{k}}\right|^{p-2} \frac{\partial u}{\partial x_{k}} \frac{\partial v}{\partial x_{k}} \\
& +\int_{G} \beta(x)|u(x)|^{p-2} u(x) v(x) d x
\end{aligned}
$$

where $|u|$ is the Euclidean norm of $u \in R^{N}$, or functionals of the type

$$
\begin{aligned}
a(u, v) \equiv & \sum_{k=1}^{N} \int_{G} \alpha(x)|\nabla u(x)|^{p-2} \frac{\partial u}{\partial x_{k}} \frac{\partial v}{\partial x_{k}} \\
& +\int_{G} \beta(x)|u(x)|^{p-2} u(x) v(x) d x .
\end{aligned}
$$

Such operators can be found in the paper of Kenmochi [20]. Suitable perturbations of these operators by Nemytskii-type pseudo-monotone operators provide us with other classes of examples where our theory applies.

In what follows, several relations hold a.e. on $G$ or on $\partial G$. This will be assumed without further mention. Let $\gamma$ be a maximal monotone graph in $\mathscr{R}^{2}$ with $0 \in \gamma(0)$ and let

$$
\mathscr{A}=-\sum_{i, j=1}^{n} \frac{\partial}{\partial x_{i}}\left(a_{i j} \frac{\partial}{\partial x_{j}}\right)
$$

be a uniformly elliptic operator with coefficients $a_{i j}$ lying in the space $C^{1}(\bar{G}, \mathscr{R})$ for every $i, j=1,2, \ldots, n$. With this operator we associate the maximal monotone operator $A$ in $L^{2}(G)$ defined by

$$
\begin{gathered}
D(A)=\left\{u \in H^{2}(G):-\frac{\partial u}{\partial n_{\alpha}} \in \gamma(u) \text { on } \partial G\right\}, \\
A u=\mathscr{A} u, u \in D(A) .
\end{gathered}
$$

Here, $\frac{\partial u}{\partial n_{\alpha}}$ is the outward normal derivative given by

$$
\frac{\partial u}{\partial n_{\alpha}}=\sum_{i, j=1}^{n} a_{i j} \frac{\partial u}{\partial x_{j}} \cos \left(\bar{n}, x_{i}\right),
$$

where $\bar{n}$ is the outward normal to $\partial G$.

For the operator $B$ we assume that

$$
(B u)(x)=g_{1}(u(x))+g_{2}(x, u(x))+\beta\left(\|u\|_{L^{2}}\right),
$$

where $g_{1}$ is as in Gupta and Hess [14], $\beta \in \Gamma$ is continuous and $g_{2}$ satisfies the following conditions:

(i) $g_{2}: \bar{G} \times \mathscr{R} \rightarrow \mathscr{R}$ is continuous and there exist positive constants $c, d$ such that

$$
\left|g_{2}(x, t)\right| \leq c+d|t|, \quad(x, t) \in G \times \mathscr{R} ;
$$


(ii) for every $u_{0} \in D(A)$ there exist $K\left(u_{0}\right)>0$ and $T=T\left(u_{0}\right)>0$ such that

$$
\int_{\{x \in G:|u(x)|>T\}} g_{2}(x, u(x))\left(u(x)-u_{0}(x)\right) d x \geq-K\left(u_{0}\right)
$$

for all $u \in D(A)$ with sufficiently large $\|u\|$.

Then, defining the operators $B_{1}(u), B_{2}(u)$ by

$$
\left(B_{1} u\right)(x)=g_{1}(u(x))
$$

and

$$
\left(B_{2} u\right)(x)=g_{2}(x, u(x))+\beta\left(\|u\|_{L^{2}}\right), \quad x \in G, u \in L^{2}(G),
$$

respectively, we have, by Theorem 3.6, that $R\left(A+B_{1}\right) \subset \overline{R\left(A+B_{1}+B_{2}\right)}$ and int $R\left(A+B_{1}\right) \subset$ int $R\left(A+B_{1}+B_{2}\right)$.

As in Gupta and Hess [14], the operator $A_{1} \equiv A+B_{1}: L^{2}(G) \supset D(A) \rightarrow 2^{L^{2}(G)}$ is maximal monotone. Also, the operator $B_{2}: L^{2}(G) \rightarrow L^{2}(G)$ is continuous and bounded by well-known facts on Nemytskii operators. Gupta and Hess showed in [14] that the operator $A_{1}$ is actually boundedly inversely compact, i.e., for every bounded $Q \subset X$ and every bounded $Q^{*} \subset X^{*}$ the set $Q \cap A_{1}^{-1}\left(Q^{*}\right)$ is relatively compact in $X$. This property was then used in [14] to show that the operator $\left(A_{1}+J\right)^{-1}$ maps bounded sets into relatively compact sets. However, as we have shown in Lemma 3.1, this operator is also continuous because the space $L^{2}(G)$ is uniformly convex. Thus, $\left(A_{1}+J\right)^{-1}$ is a compact resolvent. In order to apply Theorem 3.6, we need to show that for every $u_{0} \in D(A)$ there exist $\widetilde{K}\left(u_{0}\right)>0$ and $\widetilde{\beta}=\widetilde{\beta}_{u_{0}} \in \Gamma$ such that

$$
\left(B_{2} u, u-u_{0}\right) \geq-\tilde{K}\left(u_{0}\right)-\tilde{\beta}\left(\|u\|_{L^{2}}\right)\|u\|_{L^{2}}
$$

for all $u \in D(A)$ with sufficiently large $\|u\|_{L^{2}}$. To this end, let $u_{0} \in D(A)$ be given. Then we have

$$
\begin{aligned}
\left(B_{2} u, u-u_{0}\right)= & \int_{G}\left(g_{2}(x, u(x))+\beta\left(\|u\|_{L^{2}}\right)\right)\left(u(x)-u_{0}(x)\right) d x \\
= & \int_{G} g_{2}(x, u(x))\left(u(x)-u_{0}(x)\right) d x \\
& +\int_{G} \beta\left(\|u\|_{L^{2}}\right)\left(u(x)-u_{0}(x)\right) d x \\
\equiv & I_{1}+I_{2} .
\end{aligned}
$$

For $I_{2}$ we have

$$
\begin{aligned}
I_{2} & \geq-\beta\left(\|u\|_{L^{2}}\right) \int_{G}|u(x)| d x-\beta\left(\|u\|_{L^{2}}\right) \int_{G}\left|u_{0}(x)\right| d x \\
& \geq-\beta\left(\|u\|_{L^{2}}\right)\|u\|_{L^{2}}[\operatorname{meas}(G)]^{1 / 2}-\beta\left(\|u\|_{L^{2}}\right)\left\|u_{0}\right\|_{L^{2}}[\operatorname{meas}(G)]^{1 / 2} \\
& \geq-2 \beta\left(\|u\|_{L^{2}}\right)\|u\|_{L^{2}}[\operatorname{meas}(G)]^{1 / 2}=-\widetilde{\beta}\left(\|u\|_{L^{2}}\right)\|u\|_{L^{2}},
\end{aligned}
$$

with $u \in D(A)$ with $\|u\|_{L^{2}} \geq\left\|u_{0}\right\|_{L^{2}}$, where $\tilde{\beta} \in \Gamma$ is an obvious function. 
For $I_{1}$ we have

$$
\begin{aligned}
I_{1}= & \int_{\{x \in G:|u(x)| \leq T\}} g_{2}(x, u(x))\left(u(x)-u_{0}(x)\right) d x \\
& +\int_{\{x \in G:|u(x)|>T\}} g_{2}(x, u(x))\left(u(x)-u_{0}(x)\right) d x \\
\geq & \int_{\{x \in G:|u(x)| \leq T\}} g_{2}(x, u(x))\left(u(x)-u_{0}(x)\right) d x-K\left(u_{0}\right) \\
= & \int_{\{x \in G:|u(x)| \leq T\}} g_{2}(x, u(x)) u(x) d x \\
& -\int_{\{x \in G:|u(x)| \leq T\}} g_{2}(x, u(x)) u_{0}(x) d x-K\left(u_{0}\right) \\
\geq & -L T \operatorname{meas}(G)-L\left\|u_{0}\right\|_{L^{2}}[\operatorname{meas}(G)]^{1 / 2}-K\left(u_{0}\right) \\
= & -\widetilde{K}\left(u_{0}\right),
\end{aligned}
$$

where

$$
L=\max _{\{x \in G,|t| \leq T\}}\left\{\left|g_{2}(x, t)\right|\right\}
$$

and $\widetilde{K}\left(u_{0}\right)$ is an obvious constant. Thus our conclusion is true.

In order to show that $(5.1)$ is actually satisfied by the operator $B_{2}$ in the example of [14], we recall that in that example $\left(B_{2} u\right)(x)=g_{2}(u(x))$, where $g_{2}$ is as in (i) above and such that $g_{2}(t) t \geq 0$ for $|t| \geq T$. We have

$$
\begin{aligned}
\int_{\{x \in G:|u(x)|>T\}} & g_{2}(u(x))\left(u(x)-u_{0}(x)\right) d x \\
= & \int_{\{x \in G:|u(x)|>T\}} g_{2}(u(x)) u(x) d x \\
& -\int_{\{x \in G:|u(x)|>T\}} g_{2}(u(x)) u_{0}(x) d x \\
\geq & (1 / d)\left\|B_{2} u\right\|_{L^{2}}^{2}-(c / d)[\operatorname{meas}(G)]^{1 / 2}\left\|B_{2} u\right\|_{L^{2}}-k \\
& -\left\|B_{2} u\right\|_{L^{2}}\left\|u_{0}\right\|_{L^{2}} \\
= & {\left[(1 /(d M))\left\|B_{2} u\right\|_{L^{2}}^{2}-\left\|B_{2} u\right\|-(k / M)\right] M, }
\end{aligned}
$$

where

$$
M=\left[(c / d)[\operatorname{meas}(G)]^{1 / 2}+\left\|u_{0}\right\|_{L^{2}}\right]
$$

and $k$ is an upper bound for

$$
\frac{1}{d} \int_{\{x \in G:|u(x)| \leq T\}} g_{2}^{2}(u(x)) d x
$$

depending only on $T$. Here we have used the estimates from page 312 of [14]. Since for each $\epsilon>0$ there exists $C(\epsilon)>0$ such that $p \leq \epsilon p^{2}+C(\epsilon), p \epsilon$ $\mathscr{R}$, we conclude that there exists a constant $C=C(1 /(d M))=C\left(\left\|u_{0}\right\|_{L^{2}}\right)$ such that the last member of $(5.2)$ is bounded below by

$$
\left[-C\left(\left\|u_{0}\right\|_{L^{2}}\right)-(k / M)\right] M=-K\left(u_{0}\right),
$$


where $K\left(u_{0}\right)$ is an obvious constant. Our example is thus an improvement of the example of Gupta and Hess in [14] because the Nemytskii operator $g_{2}$ depends also on $x$ and the operator $B_{2}$ is more general in that the condition (ii) above is more general than the condition $g_{2}(t) t \geq 0,|t| \geq T$. Note that we also conclude that $R\left(A+B_{1}\right) \subset \overline{R\left(A+B_{1}+B_{2}\right)}$.

As we mentioned in Section 3 , the coercivity condition in the paper of Gupta and Hess [14] is considerably stronger than the one of Theorem 3.6. In fact, the condition in [14] reads as follows: for every $k>0$ there exists a constant $C(k) \in \mathscr{R}$ such that

$$
(B x, x) \geq k\|B x\|-C(k), \quad x \in X,
$$

where $B: X \rightarrow X^{*}$ is demicontinuous and bounded. To show that this condition implies the analogous condition of Theorem 3.6, assume that $B$ satisfies this condition and fix $x_{0} \in D(A)$. Then we have

$$
\left(B x, x-x_{0}\right)=(B x, x)-\left(B x, x_{0}\right) \geq(B x, x)-\|B x\|\left\|x_{0}\right\| .
$$

Given the number $k=\left\|x_{0}\right\|$, there exists a constant $K\left(x_{0}\right)=C\left(\left\|x_{0}\right\|\right)$ such that

$$
(B x, x) \geq\left\|x_{0}\right\|\|B x\|-K\left(x_{0}\right), \quad x \in X .
$$

Combining this with (5.3), we get

$$
(B x, x) \geq-K\left(x_{0}\right), \quad x \in X,
$$

which shows our assertion.

One of the things this paper illustrates is the strong connection between the theory of zeros and the theory of ranges of sums of nonlinear operators. It is rather natural to ask whether such connections also exist with the eigenvalue problem involving perturbations of nonlinear maximal monotone and $\mathrm{m}$ accretive operators. For example, given a set $S \subset X^{*}$, under what conditions can we conclude that there exists a number $\lambda>0$ such that $S \subset \overline{R(A+\lambda B)}$ and/or int $S \subset R(A+\lambda B)$ ? We should note here that the same number $\lambda$ should work for all points in the set $S$. As far as the authors know, this is a new direction in the spectral theory of nonlinear operators and the study of such problems will be undertaken in their future investigations.

\section{REFERENCES}

1. V. Barbu, Nonlinear Semigroups and Differential Equations in Banach Spaces, Noordhoff, Leyden, The Netherlands, 1975.

2. H. Brézis, Opérateurs maximaux monotones et semi-groupes de contractions dans les espaces de Hilbert, Math. Studies 5, North-Holland, Amsterdam, 1973.

3. of Mathematicians, Canadian Mathematical Congress, Vancouver, 1974.

4. H. Brézis and A. Haraux, Image d'une somme d'opérateurs monotones et applications, Israel J. Math. 23 (1976), 165-186.

5. H. Brézis and L. Nirenberg, Characterizations of the ranges of some nonlinear operators and applications to boundary value problems, Ann. Scuola Norm. Sup. Pisa 5 (1978), 225-326.

6. F. E. Browder, Nonlinear operators and nonlinear equations of evolution in Banach spaces, Proc. Sympos. Pure Math., vol 18, Amer. Math. Soc., Providence, RI, 1976.

7. __ On a principle of H. Brézis and its applications, J. Funct. Anal. 25 (1977), 356-365. 
8. B. D. Calvert and C. P. Gupta, Nonlinear elliptic boundary value problems in $L^{p}$ spaces and sums of ranges of accretive operators, Nonlinear Anal. TMA 2 (1978), 1-26.

9. I. Cioranescu, Geometry of Banach spaces, duality mappings and nonlinear problems, Kluwer, Boston, 1990.

10. Z. Guan, Solvability of semilinear equations with compact perturbations of operators of monotone type, Proc. Amer. Math. Soc. 121 (1994), 93-102.

11. Existence of solutions of nonlinear equations involving compact perturbations of monotone operators, Proc. First World Congress of Nonlinear Analysts, Tampa, Florida, 1992, De Gruyter (to appear).

12. Z. Guan and A. G. Kartsatos, Solvability of nonlinear equations with coercivity generated by compact perturbations of m-accretive operators in Banach spaces, Houston J. Math. (to appear).

13. C. P. Gupta, Sums of ranges of operators and applications, Nonlinear Systems and Applications, Academic Press, New York, 1977.

14. C. P. Gupta and P. Hess, Existence theorems for nonlinear noncoercive operator equations and nonlinear elliptic boundary value problems, J. Differential Equations 22 (1976), 305-313.

15. A. G. Kartsatos, Mapping theorems involving ranges of sums of nonlinear operators, Nonlinear Anal. TMA 6 (1982), 271-278.

16. __ Recent results involving compact perturbations and compact resolvents of accretive operators in Banach spaces, Proc. First World Congress of Nonlinear Analysts, Tampa, Florida, 1992, De Gruyter (to appear).

17. _ On compact perturbations and compact resolvents of nonlinear m-accretive operators in Banach spaces, Proc. Amer. Math. Soc. 119 (1993), 1189-1199.

18. _ Sets in the ranges of sums for perturbations of nonlinear m-accretive operators in Banach spaces, Proc. Amer. Math. Soc. 123 (1995), 145-156.

19. _ - Sets in the ranges of nonlinear accretive operators in Banach spaces, Studia Math. (to appear).

20. N. Kenmochi, Pseudomonotone operators and nonlinear elliptic boundary value problems, J. Math. Soc. Japan 27 (1975), 121-149.

21. V. Lakshmikantham and S. Leela, Nonlinear differential equations in abstract spaces, Pergamon Press, Oxford, 1981.

22. M. Nagumo, Degree of mapping in convex linear topological spaces, Amer. J. Math. 73 (1951), 497-511.

23. D. Pascali and Sburlan, Nonlinear mappings of monotone type, Sijthoff and Noordhoof, Bucharest, 1978.

24. J. Prüß, A characterization of uniform convexity and applications to accretive operators, Hiroshima Math. J. 11 (1981), 229-234.

25. E. Zeidler, Nonlinear functional analysis and its applications, II/B, Springer-Verlag, New York, 1990.

Department of Mathematics, University of Wisconsin-Eau Claire, Eau Claire, WisCONSIN 54702

E-mail address: guanz@uwec

Department of Mathematics, University of South Florida, Tampa, Florida 33620-5700

E-mail address: hermes@gauss . math .usf .edu 